\title{
Local Discontinuous Galerkin Method for Nonlinear Time-Space Fractional Subdiffusion/Superdiffusion Equations
}

\author{
Meilan Qiu $\left(\mathbb{D},{ }^{1}\right.$ Dewang $\mathrm{Li}\left(\mathbb{D},{ }^{1}\right.$ and Yanyun $\mathrm{Wu} \mathbb{( D}^{2}$ \\ ${ }^{1}$ School of Mathematics and Statistics, Huizhou University, Huizhou, Guangdong 516007, China \\ ${ }^{2}$ School of Science, Chongqing University of Posts and Telecommunications, Chongqing 400065, China \\ Correspondence should be addressed to Dewang Li; ldwldw1976@126.com and Yanyun Wu; wuyanyun@cqupt.edu.cn
}

Received 8 August 2019; Accepted 16 November 2019; Published 22 June 2020

Academic Editor: David González

Copyright (C) 2020 Meilan Qiu et al. This is an open access article distributed under the Creative Commons Attribution License, which permits unrestricted use, distribution, and reproduction in any medium, provided the original work is properly cited.

\begin{abstract}
Fractional partial differential equations with time-space fractional derivatives describe some important physical phenomena. For example, the subdiffusion equation (time order $0<\alpha<1$ ) is more suitable to describe the phenomena of charge carrier transport in amorphous semiconductors, nuclear magnetic resonance (NMR) diffusometry in percolative, Rouse, or reptation dynamics in polymeric systems, the diffusion of a scalar tracer in an array of convection rolls, or the dynamics of a bead in a polymeric network, and so on. However, the superdiffusion case $(1<\alpha<2)$ is more accurate to depict the special domains of rotating flows, collective slip diffusion on solid surfaces, layered velocity fields, Richardson turbulent diffusion, bulk-surface exchange controlled dynamics in porous glasses, the transport in micelle systems and heterogeneous rocks, quantum optics, single molecule spectroscopy, the transport in turbulent plasma, bacterial motion, and even for the flight of an albatross (for more physical applications of fractional sub-super diffusion equations, one can see Metzler and Klafter in 2000). In this work, we establish two fully discrete numerical schemes for solving a class of nonlinear time-space fractional subdiffusion/superdiffusion equations by using backward Euler difference $(1<\alpha<2)$ or second-order central difference $(1<\alpha<2)$ /local discontinuous Galerkin finite element mixed method. By introducing the mathematical induction method, we show the concrete analysis for the stability and the convergence rate under the $L^{2}$ norm of the two LDG schemes. In the end, we adopt several numerical experiments to validate the proposed model and demonstrate the features of the two numerical schemes, such as the optimal convergence rate in space direction is close to $\mathcal{O}\left(h^{k+1}\right)$. The convergence rate in time direction can arrive at $\mathcal{O}\left(\tau^{2-\alpha}\right)$ when the fractional derivative is $0<\alpha<1$. If the fractional derivative parameter is $1<\alpha<2$ and we choose the relationship as $h=C^{\prime} \tau$ (h denotes the space step size, $C^{\prime}$ is a constant, and $\tau$ is the time step size), then the time convergence rate can reach to $\mathcal{O}\left(\tau^{3-\alpha}\right)$. The experiment results illustrate that the proposed method is effective in solving nonlinear time-space fractional subdiffusion/superdiffusion equations.
\end{abstract}

\section{Introduction}

Over the past several decades, fractional differential equations attract more and more scholar's attention due to their wide applications in science and engineering [1-4]. Because of the nonlocality and hereditariness, as well as other properties of fractional derivatives, researchers find that fractional partial differential equations (FPDEs) can be more accurately and effectively used to depict the complex systems with memory, hereditary, and long-range interactions than classical partial differential equations. Among them, the space-time fractional diffusion equation we mean is an evolution equation; they imply for the flux fractional Fick's law which accounts for spatial and temporal nonlocality. The fundamental solution (for the Cauchy problem) of this type of fractional diffusion equation can be used to interpret a probability density evolving in time of a peculiar self-similar stochastic process, and we also view it as a generalized diffusion process [5]. Especially, fractional subdiffusion/ superdiffusion equations (FSEs) play important roles in describing a special type of anomalous diffusion process, and thus become very popular for many real applications $[6,7]$.

It is of great importance to seek efficient methods to solve fractional subdiffusion/superdiffusion equations (FSEs). However, generally speaking, it is difficult to obtain the analytic solutions for most of the fractional differential 
equations. Moreover, most nonlinear fractional subdiffusion/superdiffusion equations (FSEs) are not solvable by analytic methods. This motivates researchers to resort to efficient and reliable numerical methods for solving these fractional subdiffusion/superdiffusion equations and then understand the behaviors of them.

In recent years, a large number of researchers focus on various numerical methods for solving FSEs. For example, Zeng et al. [8] used the fast finite difference method for solving the high-dimensional time-fractional subdiffusion equation. Gao and Sun [9] applied the $L_{1}$ approximation for the time-fractional derivative and developed a compact finite difference scheme for the fractional subdiffusion equation by using the energy method; they prove the solvability and stability, as well as $L^{\infty}$ convergence. Zhang et al. presented a Crank-Nicolson-type difference scheme for solving the subdiffusion equation [10]. Wang and Vong [11] established a compact finite difference scheme for solving the modified anomalous fractional subdiffusion equation and obtained a second-order time accuracy convergence rate. Jin et al. [12] obtained a fully discrete scheme for the subdiffusion equation by developing essential initial corrections at the starting two steps for the Crank-Nicolson scheme in time and the Galerkin finite element method in space. Shen et al. in [13] applied the implicit and explicit difference methods to solve the space-time fractional convection-subdiffusion equations. Saadatmandi et al. [14] constructed a sinc-Legendre collocation method for solving the fractional convection-subdiffusion equation. Liu's group [15] analyzed a radial basis function approximation scheme for solving the fractional mobile/immobile convection-subdiffusion equation. Zhang et al. [10] proposed the two-order Crank-Nicolson difference method for solving the subdiffusion equation, and they proved the discrete $H_{1}$ norm convergence rate as well as the maximum norm error estimate. In [16], Jiang and Ma adopted the high-order finite element method to solve the timefractional subdiffusion equation. Zeng et al. [17] proposed a finite difference/element mixed approach to solve the timefractional subdiffusion problem. $\mathrm{Hu}$ et al. in [18], $\mathrm{Hu}$ et al. established the center box approach to solve the time-fractional subdiffusion system. In [19], Bhrawy et al. gave out the Legendre spectral collocation scheme for solving nonlinear fractional subdiffusion and reaction subdiffusion equations. Du et al. [20] developed a fully discrete local discontinuous Galerkin (LDG) method for solving nonlinear time-fractional fourth-order partial differential equations. Aboelenen in [21] constructed a local discontinuous Galerkin (LDG) method for solving the distributed-order time and Riesz space fractional convection-subdiffusion- and Schrödinger-type equations. Liu et al. [22] established a high-order local discontinuous Galerkin (LDG) approach combined with weighted and shifted Grünwald difference (WSGD) approximation for solving a Caputo-type time-fractional subdiffusion equation.

However, to the best of our knowledge so far, the LDG method have not been considered in solving the nonlinear time-space fractional subdiffusion and/or superdiffusion equations. Therefore, our aim in this paper is to establish two fully discrete LDG schemes to solve the nonlinear time-space fractional subdiffusion $(0<\alpha<1)$ equation:

$$
\begin{cases}\frac{\partial^{\alpha} u(x, t)}{\partial t^{\alpha}}+\frac{\partial}{\partial x} f(u)=\varepsilon\left(-(-\Delta)^{\beta / 2}\right) u(x, t), & x \in \Omega=[a, b], t \in(0, T], \\ u(x, t)=0, & x \in \partial \Omega, t \in(0, T], \\ u(x, 0)=u_{0}(x), & x \in \Omega=[a, b],\end{cases}
$$

and the nonlinear time-space fractional superdiffusion $(1<\alpha<2)$ equation:

$$
\begin{cases}\frac{\partial^{\alpha} u(x, t)}{\partial t^{\alpha}}+\frac{\partial}{\partial x} f(u)=\varepsilon\left(-(-\Delta)^{\beta / 2}\right) u(x, t), & x \in \Omega=[a, b], t \in(0, T], \\ u(x, t)=0, & x \in \partial \Omega, t \in(0, T], \\ u(x, 0)=\phi(x), & x \in \Omega=[a, b], \\ u_{t}(x, 0)=\psi(x), & x \in \Omega=[a, b],\end{cases}
$$


where $\Omega=(a, b), T>0,1<\beta<2$, denotes the order number of space fractional derivatives, $\epsilon$ is a real positive parameter, $u_{0}, \phi(x), \psi(x) \in H_{0}^{1}(\Omega)$ are the given smooth functions, and the nonlinear term $f(u)$ satisfies the Lipschitz continuous condition on the domain $\Omega$.

For simplicity, we only consider the periodic boundary conditions in this article. Notice that this assumption is not essential, and our method can be directly extended to the problems with aperiodic boundary conditions.

The time fractional derivative in nonlinear time-space fractional subdiffusion equation (1) uses the following Caputo fractional derivative $[2,3]$ :

$$
\frac{\partial^{\alpha} u(x, t)}{\partial t^{\alpha}}= \begin{cases}\frac{1}{\Gamma(1-\alpha)} \int_{0}^{t} \frac{\partial u(x, s)}{\partial s} \frac{\mathrm{d} s}{(t-s)^{\alpha}}, & 0<\alpha<1, \\ \frac{\partial u(x, t)}{\partial t}, & \alpha=1,\end{cases}
$$

and the time-fractional Caputo derivative in nonlinear timespace fractional superdiffusion equation (2) is defined as follows [23]:

$$
\frac{\partial^{\alpha} u(x, t)}{\partial t^{\alpha}}= \begin{cases}\frac{1}{\Gamma(2-\alpha)} \int_{0}^{t}(t-s)^{1-\alpha} \frac{\partial^{2} u(x, s)}{\partial s^{2}} \mathrm{~d} s, & 1<\alpha<2, \\ \frac{\partial^{2} u(x, t)}{\partial t^{2}}, & \alpha=2 .\end{cases}
$$

The spatial fractional derivative in both equations (1) and (2) is an important tool to describe the anomalous diffusion phenomenon [24]; when $1<\beta<2$, it represents a Lévy $\beta$-stable flight [25], and when $\beta \longrightarrow 2$, it depicts a Brownian diffusion process. In this paper, we still use the definition of the fractional Laplacian operator $\left(-(-\Delta)^{\beta / 2}\right) u(x, t)$, $1<\beta<2$, which is also known as the Riesz fractional derivative $\left(\partial^{\beta} / \partial|x|^{\beta}\right) u(x, t)$, and we assume that the unknown function and all its derivatives $u(x, t), u^{\prime}(x, t), \ldots$, $u^{(n-1)}(x, t)$ vanish at the end points on an infinite domain (i.e., $x=\mp \infty)$ [26].

The discontinuous Galerkin (DG) method is a classical finite element method using discontinuous, piecewise polynomials as the solution and the test spaces in the spatial direction. There are various DG methods suggested in the literature to solve diffusion problems; its generalization calls the local discontinuous Galerkin (LDG) methods which are introduced in [27] by Cockburn and Shu and further studied in $[28,29]$. Recently, the LDG method is applied to solving the fractional partial differential equations [30-32]. In $[33,34]$, Xu et al. developed the LDG method for solving the time fractional diffusion equation and fractional convectiondiffusion equations, respectively.

The organization of this article is as follows. In the second part, we review the appropriate functional spaces and some important definitions and properties of fractional derivatives. Based on the technique introduced in [34], we rewrite the fractional Laplacian operator (with the order $\beta$ ) as a composite of an integer derivative with one order and a fractional integral with an order $2-\beta$, and we, respectively, get the fully discrete LDG schemes for the nonlinear timespace fractional subdiffusion equation and the nonlinear time-space fractional superdiffusion equation in the third part. In the fourth and fifth part, we provide the concrete stability and $L^{2}$ error analysis for the two fully discrete schemes, respectively. In the sixth part, we present several examples to verify the theoretical results and elaborate the concluding remarks in the end.

\section{Preliminary Results}

In order to prove the convergence rate of two fully discrete LDG numerical schemes better, we briefly list some relevant properties of fractional integrals and derivatives as well as several basic lemmas that are needed as follows; some of them have already been proved in [24]. Therefore, we ignore the detailed proof processes here.

Lemma 1 (linearity)

$$
\begin{gathered}
{ }_{a} I_{x}^{\alpha}(\lambda f(x)+\mu g(x))=\lambda_{a} I_{x}^{\alpha} f(x)+\mu_{a} I_{x}^{\alpha} g(x) . \\
{ }_{a} D_{x}^{\alpha}(\lambda f(x)+\mu g(x))=\lambda_{a} D_{x}^{\alpha} f(x)+\mu_{a} D_{x}^{\alpha} g(x) .
\end{gathered}
$$

Lemma 2 (semigroup property)

$$
{ }_{a} I_{x}^{\alpha+\beta} f(x)={ }_{a} I_{x}^{\alpha}\left({ }_{a} I_{x}^{\beta} f(x)\right)={ }_{a} I_{x}^{\beta}\left({ }_{a} I_{x}^{\alpha} f(x)\right) .
$$

These lemmas combine with definitions 2.1-2.3 in [24]; we can convert the fractional Laplacian derivative into a composite form of an integer derivative with one order and a fractional integral with an order $2-\beta(1<\beta<2)$ [34]:

$$
-(-\Delta)^{\beta / 2} u(x, t)=\frac{\partial}{\partial x}\left(\Delta_{(\beta-2) / 2} \frac{\partial u(x, t)}{\partial x}\right) \text {. }
$$

Lemma 3 (adjoint property)

$$
\left({ }_{a} I_{x}^{\alpha} u, u\right)_{\Omega}=\left(u,{ }_{x} I_{b}^{\alpha} u\right)_{\Omega} .
$$

Lemma 4 (see [35])

$$
\left({ }_{-\infty} I_{x}^{\alpha} u,\right)_{R}=\cos (\alpha \pi)|u|_{J_{L}^{-\alpha}(R)}^{2}=\cos (\alpha \pi)|u|_{J_{R}^{-\alpha}(R)}^{2} .
$$

Lemma 5 (see [36]) Let $0<\theta<2, \theta \neq 1$. Then, for any $w, v \in H_{0}^{\theta / 2}(0, X)$, then

$$
\left({ }_{0}^{R} D_{x}^{\theta} w, v\right)_{L^{2}(0, X)}=\left({ }_{0}^{R} D_{x}^{\theta / 2} w,{ }_{t}^{R} D_{X}^{\theta / 2} v\right)_{L^{2}(0, X)} .
$$

By composite form (7) of the fractional Laplacian operator and Lemmas 3-5, together with the properties of Riemann-Liouville fractional integrals, the following lemmas hold. 
Lemma 6 For all real numbers, $0<s<1$, the fractional integral satisfies the property

$$
\left(\Delta_{-s} u, u\right)_{\Omega}=|u|_{J_{L}^{-\alpha}(\Omega)}^{2}=|u|_{J_{R}^{-\alpha}(\Omega)}^{2} .
$$

Lemma 7 (fractional Poincaré-Friedrichs inequality, see [35]).

$$
\begin{array}{ll}
\|u\|_{L^{2}(\Omega)} \leq C|u|_{J_{L, 0}^{\mu}(\Omega)}, & u \in J_{L, 0}^{\mu}(\Omega), \mu \in R, \\
\|u\|_{L^{2}(\Omega)} \leq C|u|_{J_{R, 0}^{\mu}(\Omega)}, & u \in J_{R, 0}^{\mu}(\Omega), \mu \in R .
\end{array}
$$

Lemma 8 (see [34]). Suppose $u(x)$ is a smooth function defined on $\Omega \subset R . \Omega_{h}$ is a discretization of the domain with interval width $h, u_{h}(x)$ is an approximation solution of $u$ in polynomial function space $P_{h}^{k}$. For all $j, u_{h}(x) \in I_{j}$ is a polynomial of degree up to order $k$, and $(u, v)_{I_{j}}=$ $\left(u_{h}, v\right)_{I_{j}}, \forall v \in P^{k}, k$ is degree of polynomials. For all $-1<\alpha \leq 0$, we have

$$
\left\|(\Delta)_{\beta / 2} u(x)-(\Delta)_{\beta / 2} u_{h}(x)\right\|_{L^{2}(\Omega)} \leq C h^{k+1},
$$

where $C$ is a constant independent of $h$.

To prepare the analysis of the error estimates, we need the following two projection operators in one dimension $[a, b]$; denote them by $Q$, i.e., for each $j$,

$$
\int_{I_{j}}(Q \omega(x)-\omega(x)) v(x) \mathrm{d} x=0, \quad \forall v \in P^{k}\left(I_{j}\right),
$$

and special projection $\mathscr{P}^{ \pm}$, i.e., for any $j$,

$$
\begin{aligned}
& \int_{I_{j}}\left(\mathscr{P}^{+} \omega(x)-\omega(x)\right) v(x) \mathrm{d} x=0, \\
& \forall v \in P^{k-1}\left(I_{j}\right), \mathscr{P}^{+} \omega\left(x_{j-(1 / 2)}^{+}\right)=\omega\left(x_{j-(1 / 2)}\right), \\
& \int_{I_{j}}\left(\mathscr{P}^{-} \omega(x)-\omega(x)\right) v(x) \mathrm{d} x=0, \\
& \forall v \in P^{k-1}\left(I_{j}\right), \mathscr{P}^{-} \omega\left(x_{j+(1 / 2)}^{-}\right)=\omega\left(x_{j+(1 / 2)}\right) .
\end{aligned}
$$

The above two projection operators satisfy the following approximation inequality [37]:

$$
\left\|\omega^{e}\right\|+h\left\|\omega^{e}\right\|_{\infty}+h^{1 / 2}\left\|\omega^{e}\right\|_{\tau_{h}} \leq C h^{k+1},
$$

where $\omega^{e}=\mathscr{P} \omega(x)-\omega(x)$ or $\omega^{e}=\mathscr{P}^{ \pm} \omega(x)-\omega(x)$, where the constant $C$ is just depending on $\omega$ but independent on $h$ and $\tau_{h}$ denotes the set of boundary points of all elements $I_{j}$.
In this article, we use $C$ to denote the positive constant which different values from each case and apply the usual notation of norms in Sobolev spaces. Let $(\cdot, \cdot)_{D}$ denote the scalar inner product on $L^{2}(D)$, and denote the corresponding norm by $\|\cdot\|_{D}$. If $D=\Omega$, we drop $D$.

\section{Numerical Schemes}

In this part, we present two fully discrete local discontinuous Galerkin (LDG) schemes for solving the nonlinear timespace fractional subdiffusion/superdiffusion problems, respectively.

Let $\tau=T / M$ be the time mesh size; $M$ is a positive integer, $t_{n}=n \tau, n=0,1, \ldots, M$ are mesh points, and $t_{n-(1 / 2)}=\left(t_{n}+t_{n-1}\right) / 2, n=0,1, \ldots, M$ are midmesh points. The unmarked norm $\|\cdot\|$ refers to the usual $L^{2}$ norm in the whole text.

3.1. Subdiffusion Case. We use the backward Euler scheme to discrete the time fractional derivative $\left(\partial^{\alpha} u(x, t)\right) /\left(\partial t^{\alpha}\right)$ of nonlinear subdiffusion $(0<\alpha<1)$ case $(1)$ at $t_{n}$ which is as follows $[38,39]$ :

$\frac{\partial^{\alpha} u\left(x, t_{n}\right)}{\partial t^{\alpha}}=\frac{\tau^{1-\alpha}}{\Gamma(2-\alpha)} \sum_{i=0}^{n-1} b_{i} \frac{u\left(x, t_{n-i}\right)-u\left(x, t_{n-i-1}\right)}{\tau}+r^{n}(x)$.

Here, $b_{i}=(i+1)^{1-\alpha}-i^{1-\alpha}$ satisfies properties as follows:

$$
\begin{aligned}
& 1=b_{0}>b_{1}>b_{2}>\cdots>b_{n}>0, b_{n} \longrightarrow 0(n \longrightarrow \infty), \\
& \sum_{i=1}^{n}\left(b_{i-1}-b_{i}\right)+b_{n}=1,
\end{aligned}
$$

where $r^{n}(x)$ is the truncation error and satisfies the inequality $r^{n}(x) \leq C \tau^{2-\alpha}$ [38], constant $C$ depends on $u, T, \alpha$.

Now, we rewrite equation (1) as a first-order system:

$$
\begin{aligned}
\frac{\partial^{\alpha} u(x, t)}{\partial t^{\alpha}}+\frac{\partial}{\partial x} f(u)-\sqrt{\varepsilon} \frac{\partial}{\partial x} q & =0, \\
q-\Delta_{(\beta-2) / 2} p & =0, \\
p-\sqrt{\varepsilon} \frac{\partial}{\partial x} u(x, t) & =0 .
\end{aligned}
$$

Combining with equation (18), we obtain the weak formulation of system $(21)$ at $t_{n}$ : 


$$
\begin{aligned}
& \int_{\Omega} u\left(x, t_{n}\right) v \mathrm{~d} x-\alpha_{0} \int_{\Omega}\left(f\left(u\left(x, t_{n}\right)\right)\right) v_{x} \mathrm{~d} x+\alpha_{0} \sqrt{\varepsilon} \int_{\Omega} q\left(x, t_{n}\right) v_{x} \mathrm{~d} x \\
& \quad+\alpha_{0} \sum_{j=1}^{N}\left(f\left(u\left(x, t_{n}\right)\right) v_{j+(1 / 2)}^{-}-f\left(u\left(x, t_{n}\right)\right) v_{j-(1 / 2)}^{+}\right) \\
& \quad-\alpha_{0} \sqrt{\varepsilon} \sum_{j=1}^{N}\left(q\left(x, t_{n}\right) v_{j+(1 / 2)}^{-}-q\left(x, t_{n}\right) v_{j-(1 / 2)}^{+}\right) \\
& =\sum_{i=1}^{n-1}\left(b_{i-1}-b_{i}\right) \int_{\Omega} u\left(x, t_{n-i}\right) v \mathrm{~d} x+b_{n-1} \int_{\Omega} u\left(x, t_{0}\right) v \mathrm{~d} x-\alpha_{0} \int_{\Omega} r^{n}(x) v \mathrm{~d} x, \\
& \int_{\Omega} q\left(x, t_{n}\right) w \mathrm{~d} x-\int_{\Omega} \Delta_{(\beta-2) / 2} p\left(x, t_{n}\right) w \mathrm{~d} x=0, \\
& \int_{\Omega} p\left(x, t_{n}\right) z \mathrm{~d} x+\sqrt{\varepsilon}\left(\int_{\Omega} u\left(x, t_{n}\right) z_{x} \mathrm{~d} x-\sum_{j=1}^{N}\left(u\left(x, t_{n}\right) z_{j+(1 / 2)}^{-}-u\left(x, t_{n}\right) z_{j-(1 / 2)}^{+}\right)\right)=0, \\
& \int_{\Omega} u\left(x, t_{0}\right) v \mathrm{~d} x-\int_{\Omega} u_{0}(x) v \mathrm{~d} x=0,
\end{aligned}
$$

where $\alpha_{0}=\tau^{\alpha} \Gamma(2-\alpha)$.

Let $u_{h}^{n}, p_{h}^{n}, q_{h}^{n} \in V_{h}^{k}$ be the numerical solutions of $u\left(\cdot, t_{n}\right), p\left(\cdot, t_{n}\right), q\left(\cdot, t_{n}\right)$, respectively. We define the fully discrete local discontinuous Galerkin (LDG) scheme as follows: find $u_{h}^{n}, p_{h}^{n}, q_{h}^{n} \in V_{h}^{k}$ such that, for all $v, w, z \in V_{h}^{k}$, we have

$$
\begin{aligned}
& \int_{\Omega} u_{h}^{n} v \mathrm{~d} x-\alpha_{0} \int_{\Omega} f\left(u_{h}^{n}\right) v_{x} \mathrm{~d} x+\alpha_{0} \sqrt{\varepsilon} \int_{\Omega} q_{h}^{n} v_{x} \mathrm{~d} x \\
& \quad+\alpha_{0} \sum_{j=1}^{N}\left(\widehat{f}\left(u_{h}^{n}\right) v_{j+(1 / 2)}^{-}-\widehat{f}\left(u_{h}^{n}\right) v_{j-(1 / 2)}^{+}\right)-\alpha_{0} \sqrt{\varepsilon} \sum_{j=1}^{N}\left(\widehat{q_{h}^{n}} v_{j+(1 / 2)}^{-}-\widehat{q_{h}^{n}} v_{j-(1 / 2)}^{+}\right) \\
& \quad=\sum_{i=1}^{n-1}\left(b_{i-1}-b_{i}\right) \int_{\Omega} u_{h}^{n-i} v \mathrm{~d} x+b_{n-1} \int_{\Omega} u_{h}^{0} v \mathrm{~d} x \\
& \int_{\Omega} q_{h}^{n} w \mathrm{~d} x \int-\int_{\Omega} \Delta_{(\beta-2) / 2} p_{h}^{n} w \mathrm{~d} x=0 \\
& \int_{\Omega} p_{h}^{n} z \mathrm{~d} x+\sqrt{\varepsilon}\left(\int_{\Omega} u_{h}^{n} z_{x} \mathrm{~d} x-\sum_{j=1}^{N}\left(\widehat{u_{h}^{n}} z_{j+(1 / 2)}^{-}-\widehat{u_{h}^{n}} z_{j-(1 / 2)}^{+}\right)\right)=0
\end{aligned}
$$

where $\alpha_{0}=\tau^{\alpha} \Gamma(2-\alpha)$.

The "hat" terms in equation (18) in the cell boundary terms from integration by parts are the so-called "numerical fluxes" [40], which are single-valued functions defined on the edges and should be designed based on different guiding principles for different PDEs to ensure the stability of the LDG numerical scheme. Here, for the sake of concise, we take the same alternating numerical fluxes on the boundary as in [24], i.e.,

$$
\widehat{u_{h}^{n}}=u_{h}^{n-}, \widehat{q}_{h}^{n}=q_{h}^{n+} .
$$

We remark that the choice for the fluxes (25) is not unique; in fact, the crucial part is taking $u_{h}^{n}$ and $q_{h}^{n}$ from opposite sides. For the nonlinear term $\widehat{f}$, it is applicable for taking any monotonic flux. According to equation (18), we obtain a truncation error of the above LDG scheme which is $r^{n}(x)$.
3.2. Superdiffusion Case. In this part, we exploit a center difference scheme [24] to estimate the second-order timefractional Caputo derivative $\partial^{\alpha} u(x, t) / \partial t^{\alpha}$ in the nonlinear superdiffusion case $(1<\alpha<2)$; the discretization form at $t_{n}$ is as follows:

$$
\frac{\partial^{\alpha} u\left(x, t_{n}\right)}{\partial t^{\alpha}}=\frac{\tau^{2-\alpha}}{\Gamma(3-\alpha)} \sum_{i=0}^{n-1} v_{i} \frac{u\left(x, t_{n-i}\right)-u\left(x, t_{n-i-1}\right)}{\tau}+R^{n}(x) .
$$

Here, $\quad v_{i}=(i+1)^{2-\alpha}-i^{2-\alpha}, i=0,1, \ldots, n-1, \quad$ and truncation error satisfies the inequality $R^{n}(x) \leq$ $\left(C_{u} T^{3-\alpha}\right) /(\Gamma(2-\alpha)) \tau+\left(\tau^{2}\right)$ [23], $C_{u}$ is a constant which only depends on $u$.

Analogous to the nonlinear subdiffusion equation, we also convert equation (2) into first-order system (21); combined with time discretization form equation (26), we 
obtain the weak formulation for nonlinear time-space fractional equation (2) at $t_{n}$ :

$$
\begin{aligned}
& \int_{\Omega} u\left(x, t_{n}\right) v \mathrm{~d} x-\alpha_{1} \int_{\Omega} f\left(u\left(x, t_{n}\right)\right) v_{x} \mathrm{~d} x+\alpha_{1} \sqrt{\varepsilon} \int_{\Omega} q\left(x, t_{n}\right) v_{x} \mathrm{~d} x \\
& +\alpha_{1} \sum_{j=1}^{N}\left(f\left(u\left(x, t_{n}\right)\right) v_{j+(1 / 2)}^{-}-f\left(u\left(x, t_{n}\right)\right) v_{j-(1 / 2)}^{+}\right)-\alpha_{1} \sqrt{\varepsilon} \sum_{j=1}^{N}\left(q\left(x, t_{n}\right) v_{j+(1 / 2)}^{-}-q\left(x, t_{n}\right) v_{j-(1 / 2)}^{+}\right) \\
& \quad=\sum_{i=1}^{n-2}\left(-v_{i-1}+2 v_{i}-v_{i+1}\right) \int_{\Omega} u\left(x, t_{n-i-1}\right) v \mathrm{~d} x+\left(2 v_{0}-v_{1}\right) \int_{\Omega} u\left(x, t_{n-1}\right) v \mathrm{~d} x \\
& +\left(2 v_{n-1}-v_{n-2}\right) \int_{\Omega} u\left(x, t_{0}\right) v \mathrm{~d} x-v_{n-1} \int_{\Omega} u\left(x, t_{-1}\right) v \mathrm{~d} x-\alpha_{1} \int_{\Omega} R^{n}(x) v \mathrm{~d} x, \\
& \int_{\Omega} q\left(x, t_{n}\right) w \mathrm{~d} x-\int_{\Omega} \Delta(\beta-2) / 2 p\left(x, t_{n}\right) w \mathrm{~d} x=0, \\
& \int_{\Omega} p\left(x, t_{n}\right) z \mathrm{~d} x+\sqrt{\varepsilon}_{\Omega}\left(\int_{\Omega} u\left(x, t_{n}\right) z_{x} \mathrm{~d} x-\sum_{j=1}^{N}\left(u\left(x, t_{n}\right) z_{j+(1 / 2)}^{-}-u\left(x, t_{n}\right) z_{j-(1 / 2)}^{+}\right)\right)=0, \\
& \int_{\Omega} u\left(x, t_{0}\right) v \mathrm{~d} x-\int_{\Omega} \phi(x) v \mathrm{~d} x=0, \\
& \int_{\Omega} u_{t}\left(x, t_{0}\right) v \mathrm{~d} x-\int_{\Omega} \psi(x) v \mathrm{~d} x=0,
\end{aligned}
$$

where $\alpha_{1}=\tau^{\alpha} \Gamma(3-\alpha)$.

Let $u_{h}^{n}, p_{h}^{n}, q_{h}^{n} \in V_{h}^{k}$ be the approximation of discrete local discontinuous Galerkin (LDG) scheme as $u\left(\cdot, t_{n}\right), p\left(\cdot, t_{n}\right), q\left(\cdot, t_{n}\right)$, respectively. We define a fully

follows: find $u_{h}^{n}, p_{h}^{n}, q_{h}^{n} \in V_{h}^{k}$ such that $\forall v, w, z \in V_{h}^{k}$ :

$$
\begin{aligned}
& \int_{\Omega} u_{h}^{n} v \mathrm{~d} x-\alpha_{1} \int_{\Omega} f\left(u_{h}^{n}\right) v_{x} \mathrm{~d} x+\alpha_{1} \sqrt{\varepsilon} \int_{\Omega} q_{h}^{n} v_{x} \mathrm{~d} x+\alpha_{1} \sum_{j=1}^{N}\left(\widehat{f}\left(u_{h}^{n}\right) v_{j+(1 / 2)}^{-}-\widehat{f}\left(u_{h}^{n}\right) v_{j-(1 / 2)}^{+}\right)-\alpha_{1} \sqrt{\varepsilon} \sum_{j=1}^{N}\left(\widehat{q_{h}^{n}} v_{j+(1 / 2)}^{-}-\widehat{q}_{h}^{n} v_{j-(1 / 2)}^{+}\right) \\
& =\sum_{i=1}^{n-2}\left(-v_{i-1}+2 v_{i}-v_{i+1}\right) \int_{\Omega} u_{h}^{n-i-1} v \mathrm{~d} x+\left(2 v_{0}-v_{1}\right) \int_{\Omega} u_{h}^{n-1} v \mathrm{~d} x+\left(2 v_{n-1}-v_{n-2}\right) \int_{\Omega} u_{h}^{0} v d x-v_{n-1} \int_{\Omega} u_{h}^{-1} v \mathrm{~d} x, \\
& \int_{\Omega} q_{h}^{n} w \mathrm{~d} x-\int_{\Omega} \Delta_{(\beta-2) / 2} p_{h}^{n} w \mathrm{~d} x=0 \\
& \int_{\Omega} p_{h}^{n} z \mathrm{~d} x+\sqrt{\varepsilon}\left(\int_{\Omega} u_{h}^{n} z_{x} \mathrm{~d} x-\sum_{j=1}^{N}\left(\widehat{u_{h}^{n}} z_{j+(1 / 2)}^{-}-\widehat{u}_{h}^{n} z_{j-(1 / 2)}^{+}\right)\right)=0 \\
& \int_{\Omega} u_{h}^{0} v \mathrm{~d} x-\int_{\Omega} u_{0}(x) v \mathrm{~d} x=0, \\
& \int_{\Omega}\left(u_{h}^{0}\right)_{t} v \mathrm{~d} x-\int_{\Omega} \psi(x) v \mathrm{~d} x=0,
\end{aligned}
$$

where $\alpha_{1}=\tau^{\alpha} \Gamma(3-\alpha)$.

The "hat" terms in equation (28) in the cell boundary terms from integration by parts are the so-called "numerical fluxes" [40], which are single-valued functions defined on the edges. By equation (27), we know that the truncation error of the fully discrete scheme $(28)$ is $R^{n}(x)$. 
Remarks 1. Originally, we assume that $u$ has compact support and restricts the problem to the bounded domain $\Omega$. Thus, we impose homogeneous Dirichlet boundary conditions for $u \notin \Omega$.

\section{Stability}

In this part, we prove the stability for the above two fully discrete LDG schemes (18) and (28).

Theorem 1. For periodic or compactly supported boundary conditions, the fully discrete LDG scheme (18) is unconditionally stable, and the numerical solution $u_{h}^{n}$ satisfies

$$
\left\|u_{h}^{n}\right\| \leq\left\|u_{h}^{0}\right\|, n=1,2, \ldots, M .
$$

Proof. Firstly, in order to better analyze the fully discrete LDG numerical format, we introduce the following boundary term operator $\mathscr{L}$ :

$$
\begin{aligned}
\mathscr{L}(v, w, z)= & \sqrt{\varepsilon} u(a, t) z_{1 / 2}^{+}-\frac{\sqrt{\varepsilon} \mu}{h} u(b, t) v_{N+(1 / 2)}^{-} \\
& -\sqrt{\varepsilon} u(b, t) z_{N+(1 / 2)}^{-}=0 .
\end{aligned}
$$

Using the fluxes (25) and the fluxes at the boundaries combining equality (30), after a simple rearrangement of terms, the fully LDG scheme (24) becomes

$$
\begin{aligned}
& \int_{\Omega} u_{h}^{n} v \mathrm{~d} x-\alpha_{0} \int_{\Omega} f\left(u_{h}^{n}\right) v_{x} \mathrm{~d} x+\alpha_{0} \sqrt{\varepsilon} \int_{\Omega} q_{h}^{n} v_{x} \mathrm{~d} x+\sqrt{\varepsilon} \int_{\Omega} u_{h}^{n} z_{x} \mathrm{~d} x-\int_{\Omega} \Delta_{(\beta-2) / 2} p_{h}^{n} w \mathrm{~d} x \\
& \quad+\int_{\Omega} q_{h}^{n} w \mathrm{~d} x+\int_{\Omega} p_{h}^{n} z \mathrm{~d} x+\alpha_{0} \sqrt{\varepsilon} \sum_{j=1}^{N-1}\left(q_{h}^{n}\right)_{j+(1 / 2)}^{+}[v]_{j+(1 / 2)}+\sqrt{\varepsilon} \sum_{j=1}^{N-1}\left(u_{h}^{n}\right)_{j+(1 / 2)}^{-}[z]_{j+(1 / 2)} \\
& \quad-\alpha_{0} \sum_{j=1}^{N-1}\left(\hat{f}\left(u_{h}^{n}\right)\right)_{j+(1 / 2)}[v]_{j+(1 / 2)}-\alpha_{0}\left(\left(\hat{f}\left(u_{h}^{n}\right)\right)_{1 / 2} v_{1 / 2}^{+}-\left(\hat{f}\left(u_{h}^{n}\right)\right)_{N+(1 / 2)} v_{N+(1 / 2)}^{-}\right) \\
& \quad+\alpha_{0} \sqrt{\varepsilon}\left(\left(q_{h}^{n}\right)_{1 / 2}^{+} v_{1 / 2}^{+}-\left(q_{h}^{n}\right)_{N+(1 / 2)}^{-} v_{N+(1 / 2)}^{-}\right)+\frac{\sqrt{\varepsilon} \mu}{h}\left(\left(u_{h}^{n}\right)_{N+(1 / 2)}^{-}\right)^{2} \\
& =\sum_{i=1}^{n-1}\left(b_{i-1}-b_{i}\right) \int_{\Omega} u_{h}^{n-i} v \mathrm{~d} x+b_{n-1} \int_{\Omega} u_{h}^{0} v \mathrm{~d} x .
\end{aligned}
$$

Now, we prove Theorem 1 by using the mathematical induction. First, consider $n=1$; then, the LDG scheme (31) is

$$
\begin{aligned}
& \int_{\Omega} u_{h}^{1} v \mathrm{~d} x-\alpha_{0} \int_{\Omega} f\left(u_{h}^{1}\right) v_{x} \mathrm{~d} x+\alpha_{0} \sqrt{\varepsilon} \int_{\Omega} q_{h}^{1} v_{x} \mathrm{~d} x+\sqrt{\varepsilon} \int_{\Omega} u_{h}^{1} z_{x} \mathrm{~d} x-\int_{\Omega} \Delta_{(\beta-2) / 2} p_{h}^{1} w \mathrm{~d} x \\
& +\int_{\Omega} q_{h}^{1} w \mathrm{~d} x+\int_{\Omega} p_{h}^{1} z \mathrm{~d} x+\alpha_{0} \sqrt{\varepsilon} \sum_{j=1}^{N-1}\left(q_{h}^{1}\right)_{j+(1 / 2)}^{+}[v]_{j+(1 / 2)}+\sqrt{\varepsilon} \sum_{j=1}^{N-1}\left(u_{h}^{1}\right)_{j+(1 / 2)}^{-}[z]_{j+(1 / 2)} \\
& -\alpha_{0} \sum_{j=1}^{N-1}\left(\widehat{f}\left(u_{h}^{1}\right)\right)_{j+(1 / 2)}[v]_{j+(1 / 2)}-\alpha_{0}\left(\left(\widehat{f}\left(u_{h}^{1}\right)\right)_{1 / 2} v_{1 / 2}^{+}-\left(\widehat{f}\left(u_{h}^{1}\right)\right)_{N+(1 / 2)} v_{N+(1 / 2)}^{-}\right) \\
& +\alpha_{0} \sqrt{\varepsilon}\left(\left(q_{h}^{1}\right)_{1 / 2}^{+} v_{1 / 2}^{+}-\left(q_{h}^{1}\right)_{N+(1 / 2)}^{-} v_{N+(1 / 2)}^{-}\right)+\frac{\sqrt{\varepsilon} \mu}{h}\left(\left(u_{h}^{1}\right)_{N+(1 / 2)}^{-}\right)^{2}=\int_{\Omega} u_{h}^{0} v \mathrm{~d} x .
\end{aligned}
$$

Define $\Phi(u)=\int^{u} f(u) \mathrm{d} u$, and take the test functions as $v=u_{h}^{1}, w=-\alpha_{0} p_{h}^{1}, z=\alpha_{0} q_{h}^{1}$; plug them into equation (32), and we get 


$$
\begin{aligned}
& \left\|u_{h}^{1}\right\|^{2}+\alpha_{0}\left(\Delta_{(\beta-2) / 2} p_{h}^{1}, p_{h}^{1}\right)+\frac{\alpha_{0} \sqrt{\varepsilon} \mu}{h}\left(\left(u_{h}^{1}\right)_{N+(1 / 2)}^{-}\right)^{2} \\
& \quad+\alpha_{0}\left(\Phi\left(u_{h}^{1}\right)_{1 / 2}-\Phi\left(u_{h}^{1}\right)_{N+(1 / 2)}-\left(\hat{f}\left(u_{h}^{1}\right)\right)_{1 / 2}\left(u_{h}^{1}\right)_{1 / 2}^{+}+\left(\hat{f}\left(u_{h}^{1}\right)\right)_{N+(1 / 2)}\left(u_{h}^{1}\right)_{N+(1 / 2)}^{-}\right) \\
& \quad+\alpha_{0} \sum_{j=1}^{N-1}\left(\left[\Phi\left(u_{h}^{1}\right)\right]_{j+(1 / 2)}-\hat{f}\left[u_{h}^{1}\right]_{j+(1 / 2)}\right)=\int_{\Omega} u_{h}^{0} u_{h}^{1} \mathrm{~d} x,
\end{aligned}
$$

where we use the following results in the above equation:

$$
\begin{aligned}
& \int_{\Omega} f(u) u_{x} \mathrm{~d} x=\int_{\Omega} f(u) \mathrm{d} u=\int_{\Omega} \Phi^{\prime}(u) \mathrm{d} x \\
& \quad=\left.\sum_{j=1}^{N} \Phi(x)\right|_{x_{j+1 / 2)}^{+}} ^{x_{j+1 / 2)}}=\sum_{j=1}^{N}\left((\Phi(x))_{j+(1 / 2)}^{-}-(\Phi(x))_{j-(1 / 2)}^{+}\right) \\
& \quad=-\sum_{j=1}^{N-1}[\Phi(u)]_{j+(1 / 2)}-(\Phi(u))_{(1 / 2)}+(\Phi(u))_{N+(1 / 2)}, \\
& \alpha_{0} \sqrt{\varepsilon} \int_{\Omega} q_{h}^{1}\left(u_{h}^{1}\right)_{x} \mathrm{~d} x+\alpha_{0} \sqrt{\varepsilon} \int_{\Omega} u_{h}^{1}\left(q_{h}^{1}\right)_{x} \mathrm{~d} x+\alpha_{0} \sqrt{\varepsilon} \sum_{j=1}^{N-1}\left(q_{h}^{1}\right)_{j+(1 / 2)}^{+}\left[u_{h}^{1}\right]_{j+(1 / 2)}+\alpha_{0} \sqrt{\varepsilon} \sum_{j=1}^{N-1}\left(u_{h}^{1}\right)_{j+(1 / 2)}^{-}\left[q_{h}^{1}\right]_{j+(1 / 2)} \\
& \quad=\left.\alpha_{0} \sqrt{\varepsilon} \sum_{j=1}^{N}(q u)\right|_{x_{j+1 / 2}^{+}\left(x_{j}^{-}\right)}+\alpha_{0} \sqrt{\varepsilon} \sum_{j=1}^{N-1}(q)_{j+(1 / 2)}^{+}[u]_{j+(1 / 2)}+\alpha_{0} \sqrt{\varepsilon} \sum_{j=1}^{N-1} u_{j+(1 / 2)}^{-}[q]_{j+(1 / 2)} \\
& \quad=-\alpha_{0} \sqrt{\varepsilon} q_{1 / 2}^{+} u_{1 / 2}^{+}+\alpha_{0} \sqrt{\varepsilon} q_{N+(1 / 2)}^{-} u_{N+(1 / 2)}^{-}-\alpha_{0} \sqrt{\varepsilon} \sum_{j=1}^{N-1} q_{j+(1 / 2)}^{+}[u]_{j+(1 / 2)} \\
& \quad-\alpha_{0} \sqrt{\varepsilon} \sum_{j=1}^{N-1} u_{j+(1 / 2)}^{-}[q]_{j+(1 / 2)}+\alpha_{0} \sqrt{\varepsilon} \sum_{j=1}^{N-1} q_{j+(1 / 2)}^{+}[u]_{j+(1 / 2)}+\alpha_{0} \sqrt{\varepsilon} \sum_{j=1}^{N-1} u_{j+(1 / 2)}^{-}[q]_{j+(1 / 2)} \\
& \quad=-\alpha_{0} \sqrt{\varepsilon}\left(q_{1 / 2}^{+} u_{1 / 2}^{+}-q_{N+(1 / 2)}^{-} u_{N+(1 / 2)}^{-}\right) .
\end{aligned}
$$

From the properties of monotonic fluxes, we know that the function $f\left(u^{-}, u^{+}\right)$is nondecreasing with its first argument and nonincreasing with its second argument. Hence, we have $[\Phi(u)]_{j+(1 / 2)}-\widehat{f}_{h}\left[u_{h}\right]_{j+(1 / 2)}>0,1 \leq j \leq N-1$. Recall the orthogonality property of Galerkin and the following Cauchy-Schwartz inequality:

$$
\int_{\Omega} u_{h}^{0} u_{h}^{1} \mathrm{~d} x \leq \frac{1}{2}\left\|u_{h}^{1}\right\|^{2}+\frac{1}{2}\left\|u_{h}^{0}\right\|^{2} .
$$

We can rewrite equation (33) as

$$
\begin{aligned}
u_{h}^{12} & +\alpha_{0}\left(\Delta_{(\beta-2) / 2} p_{h}^{1}, p_{h}^{1}\right)+\frac{\alpha_{0} \sqrt{\epsilon} \mu}{h}\left(\left(u_{h}^{1}\right)_{N+(1 / 2)}^{-}\right)^{2} \\
& +\alpha_{0}\left(\Phi\left(u_{h}^{1}\right)_{(1 / 2)}-\Phi\left(u_{h}^{1}\right)_{N+(1 / 2)}-\left(\hat{f}\left(u_{h}^{1}\right)\right)_{\frac{1}{2}}\left(u_{h}^{1}\right)_{\frac{1}{2}}^{+}\right. \\
& \left.+\left(\hat{f}\left(u_{h}^{1}\right)\right)_{N+(1 / 2)}\left(u_{h}^{1}\right)_{N+(1 / 2)}^{-}\right) \\
\leq & \frac{1}{2}\left\|u_{h}^{1}\right\|^{2}+\frac{1}{2}\left\|u_{h}^{0}\right\|^{2} .
\end{aligned}
$$

By boundary conditions (periodic) and recalling Lemma 6 , we get

$$
u_{h}^{1} \leq\left\|u_{h}^{0}\right\| .
$$

Next, we suppose that the following inequality holds:

$$
\left\|u_{h}^{m}\right\| \leq\left\|u_{h}^{0}\right\|, \quad m=1,2, \ldots, p .
$$

Now, we need to prove $\left\|u_{h}^{p+1}\right\| \leq\left\|u_{h}^{0}\right\|$. Let $n=p+1$, and take the test functions $v=u_{h}^{p+1}, w=-\alpha_{0} p_{h}^{p+1}$, and $z=$ $\alpha_{0} q_{h}^{p+1}$ in equation (31):

$$
\begin{aligned}
& \left\|u_{h}^{p+1}\right\|^{2}+\alpha_{0}\left(\Delta_{(\beta-2) / 2} p_{h}^{p+1}, p_{h}^{p+1}\right)+\frac{\alpha_{0} \sqrt{\varepsilon} \mu}{h}\left(\left(u_{h}^{p+1}\right)_{N+(1 / 2)}^{-}\right)^{2} \\
& \quad+\alpha_{0}\left(\Phi\left(u_{h}^{p+1}\right)_{1 / 2}-\Phi\left(u_{h}^{p+1}\right)_{N+(1 / 2)}-\left(\widehat{f}\left(u_{h}^{p+1}\right)\right)_{1 / 2}\left(u_{h}^{p+1}\right)_{1 / 2}^{+}\right. \\
& \left.\quad+\left(\widehat{f}\left(u_{h}^{p+1}\right)\right)_{N+(1 / 2)}\left(u_{h}^{p+1}\right)_{N+(1 / 2)}^{-}\right) \\
& =\sum_{i=1}^{p}\left(b_{i-1}-b_{i}\right) \int_{\Omega} u_{h}^{p+1-i} u_{h}^{p+1} \mathrm{~d} x+b_{p} \int_{\Omega} u_{h}^{0} u_{h}^{p+1} \mathrm{~d} x .
\end{aligned}
$$

Analogously, consider the properties of the monotone flux $\hat{f}$ and boundary conditions again, and we get 


$$
\begin{aligned}
& \left\|u_{h}^{p+1}\right\|^{2}+\alpha_{0}\left(\Delta_{(\beta-2) / 2} p_{h}^{p+1}, p_{h}^{p+1}\right) \\
& \leq \sum_{i=1}^{p}\left(b_{i-1}-b_{i}\right)\left\|u_{h}^{p+1-i}\right\|\left\|u_{h}^{p+1}\right\|+b_{p}\left\|u_{h}^{0}\right\|\left\|u_{h}^{p+1}\right\| \\
& \leq\left(\sum_{i=1}^{p}\left(b_{i-1}-b_{i}\right)+b_{p}\right)\left\|u_{h}^{0}\right\|\left\|u_{h}^{p+1}\right\| \\
& \leq\left\|u_{h}^{0}\right\|\left\|u_{h}^{p+1}\right\| .
\end{aligned}
$$

Combining with Lemma 6, we know that

$$
\alpha_{0}\left(\Delta_{(\beta-2) / 2} p_{h}^{p+1}, p_{h}^{p+1}\right)=\alpha_{0}\left|p_{h}^{p+1}\right|_{L}^{2} J_{(\Omega)}^{(\beta-2) / 2} \geq 0
$$

Then, the last inequality in (40) gives

$$
\left\|u_{h}^{p+1}\right\| \leq\left\|u_{h}^{0}\right\| .
$$

Theorem 2. For a sufficiently small step size, $0<\tau<T$, the fully discrete LDG scheme (28) is unconditional stable, and the numerical solution $u_{h}^{n}$ satisfies

$$
\left\|u_{h}^{n}\right\| \leq C(\|\phi(x)\|+\tau\|\psi(x)\|), \quad n=0,1, \ldots, M .
$$

Proof. Similar to the nonlinear subdiffusion case, by using the inequality $\left\|u^{-1}\right\| \leq C(\|\phi(x)\|+\tau \psi(x))$ and adopting the same methods and techniques as in Theorem 1, we can obtain the conclusions of Theorem 2. For brevity, we ignore the details of proof process here.

\section{Error Estimation}

Theorem 3. Let $u\left(x, t_{n}\right)$ be the exact solution of nonlinear subdiffusion problem (1) which is sufficiently smooth with bounded derivatives, and assume that $f \in C^{3}$; let $u_{h}^{n}$ be numerical solutions of the LDG scheme (24), and we adopt $e_{u}=u\left(x, t_{n}\right)-u_{h}^{n}$ to denote the corresponding numerical error; then, there hold the following error estimates:

When $0<\alpha<1$,

$$
\left\|u\left(x, t_{n}\right)-u_{h}^{n}\right\| \leq \frac{C_{u} T^{\alpha}}{1-\alpha}\left(\tau^{-\alpha} h^{k+1}+\tau^{2-\alpha}+\tau^{-\alpha / 2} h^{k+1 / 2}+h^{k+1}\right),
$$

and when $\alpha \longrightarrow 1$,

$$
\left\|u\left(x, t_{n}\right)-u_{h}^{n}\right\| \leq C_{u} T\left(\tau^{-1} h^{k+1}+\tau+\tau^{-1 / 2} h^{k+1 / 2}+h^{k+1}\right),
$$

where $C_{u}$ is a constant which only depends on $u$.

Proof. Denote

$$
\begin{aligned}
e_{u}^{n} & =u\left(x, t_{n}\right)-u_{h}^{n}=\mathscr{P}^{-} e_{u}^{n}-\left(\mathscr{P}^{-} u\left(x, t_{n}\right)-u\left(x, t_{n}\right)\right), \\
e_{p}^{n} & =p\left(x, t_{n}\right)-p_{h}^{n}=\mathscr{Q} e_{p}^{n}-\left(\mathscr{Q} p\left(x, t_{n}\right)-p\left(x, t_{n}\right)\right), \\
e_{q}^{n} & =q\left(x, t_{n}\right)-q_{h}^{n}=\mathscr{P}^{+} e_{q}^{n}-\left(\mathscr{P}^{+} q\left(x, t_{n}\right)-q\left(x, t_{n}\right)\right) .
\end{aligned}
$$

By subtracting equation (24) from equation (23) and combining with the fluxes on boundaries, we obtain the error equation:

$$
\begin{aligned}
& \int_{\Omega} e_{u}^{n} v \mathrm{~d} x+\alpha_{0} \sqrt{\varepsilon}\left(\int_{\Omega} e_{q}^{n} v_{x} \mathrm{~d} x-\sum_{j=1}^{N}\left(\left(e_{q}^{n}\right)^{+} v_{j+(1 / 2)}^{-}-\left(e_{q}^{n}\right)^{+} v_{j-(1 / 2)}^{+}\right)\right) \\
& -\alpha_{0} \int_{\Omega}\left(f\left(u\left(x, t_{n}\right)\right)-f\left(u_{h}^{n}\right)\right) v_{x} \mathrm{~d} x+\alpha_{0} \sum_{j=1}^{N}\left(f\left(u\left(x, t_{n}\right)\right)-\widehat{f}\left(u_{h}^{n}\right)\right) v_{j+(1 / 2)}^{-} \\
& -\alpha_{0} \sum_{j=1}^{N}\left(f\left(u\left(x, t_{n}\right)\right)-\hat{f}\left(u_{h}^{n}\right)\right) v_{j-(1 / 2)}^{+}-\sum_{i=1}^{n-1}\left(b_{i-1}-b_{i}\right) \int_{\Omega} e_{u}^{n-i} v \mathrm{~d} x \\
& -b_{n-1} \int_{\Omega} e_{u}^{0} v \mathrm{~d} x+\alpha_{0} \int_{\Omega} r^{n}(x) v \mathrm{~d} x+\int_{\Omega} e_{q}^{n} w \mathrm{~d} x-\int_{\Omega} \Delta_{(\beta-2) / 2} e_{p}^{n} w \mathrm{~d} x \\
& +\int_{\Omega} e_{p}^{n} z \mathrm{~d} x+\sqrt{\varepsilon}\left(\int_{\Omega} e_{u}^{n} z_{x} \mathrm{~d} x-\sum_{j=1}^{N}\left(\left(e_{u}^{n}\right)^{-} z_{j+(1 / 2)}^{-}-\left(e_{u}^{n}\right)^{-} z_{j-(1 / 2)}^{+}\right)\right)=0,
\end{aligned}
$$


for all $v, w, z \in V_{h}^{k}$.

Define

$$
\begin{aligned}
\mathscr{F}\left(f: u, u_{h}: v\right)= & \alpha_{0} \int_{\Omega}\left(f\left(u\left(x, t_{n}\right)\right)-f\left(u_{h}^{n}\right)\right) v_{x} \mathrm{~d} x \\
& -\alpha_{0} \sum_{j=1}^{N}\left(f\left(u\left(x, t_{n}\right)\right)-\widehat{f}\left(u_{h}^{n}\right)\right) v_{j+(1 / 2)}^{-} \\
& +\alpha_{0} \sum_{j=1}^{N}\left(f\left(u\left(x, t_{n}\right)\right)-\widehat{f}\left(u_{h}^{n}\right)\right) v_{j-(1 / 2)}^{+} .
\end{aligned}
$$

Together with equations (46) and (30), after a simple rearrangement of terms, we can rewrite error equation (47) as

$$
\begin{aligned}
& \int_{\Omega} \mathscr{P}^{-} e_{u}^{n} v \mathrm{~d} x+\alpha_{0} \sqrt{\varepsilon} \int_{\Omega} \mathscr{P}^{+} e_{q}^{n} v_{x} \mathrm{~d} x+\sqrt{\varepsilon} \int_{\Omega} \mathscr{P}^{-} e_{u}^{n} z_{x} \mathrm{~d} x-\int_{\Omega} \Delta_{(\beta-2) / 2} Q e_{p}^{n} w \mathrm{~d} x \\
& \quad+\int_{\Omega} \mathscr{P}^{+} e_{q}^{n} w \mathrm{~d} x+\int_{\Omega} \mathscr{Q} e_{p}^{n} z \mathrm{~d} x+\alpha_{0} \sqrt{\varepsilon} \sum_{j=1}^{N-1}\left(\mathscr{P}^{+} e_{q}^{n}\right)_{j+(1 / 2)}^{+}[v]_{j+(1 / 2)}+\sqrt{\varepsilon} \sum_{j=1}^{N-1}\left(\mathscr{P}^{-} e_{u}^{n}\right)_{j+(1 / 2)}^{-} \\
& \quad \times[z]_{j+(1 / 2)}+\alpha_{0} \sqrt{\varepsilon}\left(\mathscr{P}^{+} e_{q}^{n}\right)_{1 / 2}^{+} v_{1 / 2}^{+}-\alpha_{0} \sqrt{\varepsilon}\left(\mathscr{P}^{+} e_{q}^{n}\right)_{N+(1 / 2)}^{-} v_{N+(1 / 2)}^{-}+\frac{\sqrt{\varepsilon} \mu}{h}\left(\mathscr{P}^{-} e_{u}^{n}\right)_{N+(1 / 2)}^{-} v_{N+(1 / 2)}^{-} \\
& =\sum_{i=1}^{n-1}\left(b_{i-1}-b_{i}\right) \int_{\Omega} \mathscr{P}^{-} e_{u}^{n-i} v \mathrm{~d} x+b_{n-1} \int_{\Omega} \mathscr{P}^{-} e_{u}^{0} v \mathrm{~d} x-\alpha_{0} \int_{\Omega} r^{n}(x) v \mathrm{~d} x \\
& \quad+\int_{\Omega}\left(\mathscr{P}^{-} u\left(x, t_{n}\right)-u\left(x, t_{n}\right)\right) v \mathrm{~d} x+\alpha_{0} \sqrt{\varepsilon} \int_{\Omega}\left(\mathscr{P}^{+} q\left(x, t_{n}\right)-q\left(x, t_{n}\right)\right) v_{x} \mathrm{~d} x \\
& \quad+\sqrt{\varepsilon} \int_{\Omega}\left(\mathscr{P}^{-} u\left(x, t_{n}\right)-u\left(x, t_{n}\right)\right) z_{x} \mathrm{~d} x-\int_{\Omega} \Delta_{(\beta-2) / 2}\left(\mathscr{Q} p\left(x, t_{n}\right)-p\left(x, t_{n}\right)\right) w \mathrm{~d} x \\
& \quad+\int_{\Omega}\left(\mathscr{P}^{+} q\left(x, t_{n}\right)-q\left(x, t_{n}\right)\right) w \mathrm{~d} x+\int_{\Omega}\left(\mathscr{Q}_{p}\left(x, t_{n}\right)-p\left(x, t_{n}\right)\right) z \mathrm{~d} x-\sum_{i=1}^{n-1}\left(b_{i-1}-b_{i}\right) \\
& \quad \times \int_{\Omega}\left(\mathscr{P}^{-} u\left(x, t_{n-i}\right)-u\left(x, t_{n-i}\right)\right) v \mathrm{~d} x-b_{n-1} \int_{\Omega}\left(\mathscr{P}^{-} u\left(x, t_{0}\right)-u\left(x, t_{0}\right)\right) v \mathrm{~d} x \\
& \quad+\alpha_{0} \sqrt{\varepsilon} \sum_{j=1}^{N-1}\left(\mathscr{P}^{+} q\left(x, t_{n}\right)-q\left(x, t_{n}\right)\right)_{j+(1 / 2)}^{+}[v]_{j+(1 / 2)}+\sqrt{\varepsilon} \sum_{j=1}^{N-1}\left(\mathscr{P}^{-} u\left(x, t_{n}\right)-u\left(x, t_{n}\right)\right)_{j+(1 / 2)}^{-} \\
& \quad \times[z]_{j+(1 / 2)}+\alpha_{0} \sqrt{\varepsilon}\left(\mathscr{P}^{+} q\left(x, t_{n}\right)-q\left(x, t_{n}\right)\right)_{1 / 2}^{+} v_{1 / 2}^{+}-\alpha_{0} \sqrt{\varepsilon}\left(\mathscr{P}^{+} q\left(x, t_{n}\right) .\right.
\end{aligned}
$$

Next, we estimate the last term on the right-hand side of energy equation (49). We redefine it as in the following form [41]:

$$
\begin{aligned}
\mathscr{F}\left(f: u, u_{h}: v\right)= & \alpha_{0} \int_{\Omega}\left(f\left(u\left(x, t_{n}\right)\right)-f\left(u_{h}^{n}\right)\right) v_{x} \mathrm{~d} x \\
& +\alpha_{0} \sum_{j=1}^{N}\left(\left(f\left(u\left(x, t_{n}\right)\right)-f\left(\bar{u}_{h}^{n}\right)\right)[v]\right)_{j+(1 / 2)} \\
& +\alpha_{0} \sum_{j=1}^{N}\left(f\left(\bar{u}_{h}^{n}\right)-\hat{f}\left(u_{h}^{n}\right)[v]\right)_{j+(1 / 2)},
\end{aligned}
$$

where $\bar{u}_{h}^{n}$ is an average value which is defined by $\bar{u}_{h}^{n}=1 / 2\left(\left(u_{h}^{n}\right)^{+}+\left(u_{h}^{n}\right)^{-}\right)$.
In order to obtain the estimation of the nonlinear term in equation (5), we need the following results:

Lemma 9 (see [42]). For each piecewise smooth function $\omega \in L^{2}(\Omega)$, we define the following function on every cell boundary point:

$$
\kappa(\widehat{f} ; \omega) \equiv \kappa\left(\widehat{f} ; \omega^{-}, \omega^{+}\right) \triangleq \begin{cases}{[\omega]^{-1}(f(\bar{\omega})-\hat{f}(\omega)),} & \text { if }[\omega] \neq 0, \\ \frac{1}{2}\left|f^{\prime}(\bar{\omega})\right|, & \text { if }[\omega]=0 .\end{cases}
$$

Here, $\hat{f}(\omega) \equiv \hat{f}\left(\omega^{-}, \omega^{+}\right)$is a monotone numerical flux, which is consistent with the given flux function $f$. And 
$\kappa(\widehat{f} ; \omega)$ is nonnegative and bounded for all $\left(\omega^{-}, \omega^{+}\right) \in R$. Moreover, we obtain

$\frac{1}{2}\left|f^{\prime}(\bar{\omega})\right| \leq \kappa(\widehat{f} ; \omega)+C_{*}|[\omega]|,-\frac{1}{8} f^{\prime \prime}(\bar{\omega})[\omega] \leq \kappa(\widehat{f} ; \omega)+C_{*}|[\omega]|^{2}$,

where the positive constant $C_{*}$ depends solely on the maximum of $\left|f^{\prime \prime}\right|$ and/or $\left|f^{\prime \prime \prime}\right|$.

In order to estimate the nonlinear term $f(u(x, t))$, we need to assume that, for sufficiently small space step size $h$ and for all $k \geq 1$, we prove that [41]

$$
\left\|e_{u}\right\|=\left\|u(x, t)-u_{h}\right\| \leq h .
$$

Lemma 10. For the operator $\mathscr{F}\left(f: u, u_{h}: v\right)$ defined in equation (50), we have the following estimate:

$$
\begin{aligned}
\mathscr{F}\left(f: u, u_{h}: v\right) \leq & -\frac{1}{4} a\left(\hat{f} ; u_{h}\right)[v]^{2} \tau^{\alpha} \\
& +\left(C+C_{*}\left(\|v\|_{\infty}+h^{-1} e_{u \infty}^{2}\right)\right)\|v\|_{L^{2}(\Omega)}^{2} \tau^{\alpha} \\
& +\left(C+C_{*} h^{-1} e_{u_{\infty}}^{2}\right) h^{2 k+1} \tau^{\alpha} .
\end{aligned}
$$

The proof of Lemma 10 is similar to Xu and Shu's work in [41]; here, we ignore the detail process of the proof.

Take the test functions as $v=\mathscr{P}^{-} e_{u}^{n}, w=-\alpha_{0} \mathscr{Q} e_{p}^{n}$, $z=\alpha_{0} \mathscr{P}^{+} e_{q}^{n}$, and plug them into equation (49); then, by using the properties (14)-(16), we get the following equality:

$$
\begin{aligned}
& \left\|\mathscr{P}^{-} e_{u}^{n}\right\|^{2}+\alpha_{0}\left(\Delta_{(\beta-2) / 2} Q e_{p}^{n}, \mathscr{Q} e_{p}^{n}\right)+\frac{\alpha_{0} \sqrt{\varepsilon} \mu}{h}\left(\mathscr{P}^{-} e_{u}^{n}\right)_{N+(1 / 2)}^{2} \\
& =\sum_{i=1}^{n-1}\left(b_{i-1}-b_{i}\right) \int_{\Omega} \mathscr{P}^{-} e_{u}^{n-i} \mathscr{P}^{-} e_{u}^{n} \mathrm{~d} x+b_{n-1} \int_{\Omega} \mathscr{P}^{-} e_{u}^{0} \mathscr{P}^{-} e_{u}^{n} \mathrm{~d} x-\alpha_{0} \int_{\Omega} r^{n}(x) \mathscr{P}^{-} e_{u}^{n} \mathrm{~d} x \\
& +\int_{\Omega}\left(\mathscr{P}^{-} u\left(x, t_{n}\right)-u\left(x, t_{n}\right)\right) \mathscr{P}^{-} e_{u}^{n} \mathrm{~d} x+\alpha_{0} \sqrt{\varepsilon} \int_{\Omega}\left(\mathscr{P}^{+} q\left(x, t_{n}\right)-q\left(x, t_{n}\right)\right)\left(\mathscr{P}^{-} e_{u}^{n}\right)_{x} \mathrm{~d} x \\
& +\alpha_{0} \sqrt{\varepsilon} \int_{\Omega}\left(\mathscr{P}^{-} u\left(x, t_{n}\right)-u\left(x, t_{n}\right)\right)\left(\mathscr{P}^{+} e_{q}^{n}\right)_{x} \mathrm{~d} x+\alpha_{0} \int_{\Omega} \Delta_{(\beta-2) / 2}\left(\mathscr{Q} p\left(x, t_{n}\right)-p\left(x, t_{n}\right)\right) \times \mathscr{Q} e_{p}^{n} \mathrm{~d} x \\
& \quad-\alpha_{0} \int_{\Omega}\left(\mathscr{P}^{+} q\left(x, t_{n}\right)-q\left(x, t_{n}\right)\right) \mathscr{Q} e_{p}^{n} \mathrm{~d} x+\alpha_{0} \int_{\Omega}\left(\mathscr{Q} p\left(x, t_{n}\right)-p\left(x, t_{n}\right)\right) \times \mathscr{P}^{+} e_{q}^{n} \mathrm{~d} x \\
& \left.\quad-\sum_{i=1}^{n-1}\left(b_{i-1}-b_{i}\right)\right]_{\Omega}\left(\mathscr{P}^{-} u\left(x, t_{n-i}\right)-u\left(x, t_{n-i}\right)\right) \mathscr{P}^{-} e_{u}^{n} \mathrm{~d} x-b_{n-1} \int_{\Omega}\left(\mathscr{P}^{-} u\left(x, t_{0}\right)-u\left(x, t_{0}\right)\right) \mathscr{P}^{-} e_{u}^{n} \mathrm{~d} x \\
& +\alpha_{0} \sqrt{\varepsilon} \sum_{j=1}^{N-1}\left(\mathscr{P}^{+} q\left(x, t_{n}\right)-q\left(x, t_{n}\right)\right)_{j+(1 / 2)}^{+}\left[\mathscr{P}^{-} e_{u}^{n}\right]_{j+(1 / 2)} \\
& +\frac{\sqrt{\varepsilon} \mu}{h}\left(\mathscr{P}^{-} u\left(x, t_{n}\right)-u\left(x, t_{n}\right)\right)_{N+(1 / 2)}^{-}\left(\left(\mathscr{P}^{-} e_{u}^{n}\right)^{-}\right)_{N+(1 / 2)}+\mathscr{F}\left(f: u, u_{h}: v\right), \\
& +\alpha_{0} \sqrt{\varepsilon} \sum_{j=1}^{N-1}\left(\mathscr{P}^{-} u\left(x, t_{n}\right)-u\left(x, t_{n}\right)\right)_{j+(1 / 2)}^{-}\left[\mathscr{P}^{+} e_{q}^{n}\right]_{j+(1 / 2)}+\alpha_{0} \sqrt{\varepsilon}\left(\mathscr{P}^{+} q\left(x, t_{n}\right)-q\left(x, t_{n}\right)\right)_{1 / 2}^{+}\left(\mathscr{P}^{-} e_{u}^{n}\right)_{1 / 2}^{+} \\
&
\end{aligned}
$$


i.e.,

$$
\begin{aligned}
& \left\|\mathscr{P}^{-} e_{u}^{n}\right\|^{2}+\alpha_{0}\left(\Delta_{(\beta-2) / 2} \mathscr{Q} e_{p}^{n}, \mathscr{Q} e_{p}^{n}\right)+\frac{\alpha_{0} \sqrt{\varepsilon} \mu}{h}\left(\mathscr{P}^{-} e_{u}^{n}\right)_{N+(1 / 2)}^{2} \\
& =\sum_{i=1}^{n-1}\left(b_{i-1}-b_{i}\right) \int_{\Omega} \mathscr{P}^{-} e_{u}^{n-i} \mathscr{P}^{-} e_{u}^{n} \mathrm{~d} x+b_{n-1} \int_{\Omega} \mathscr{P}^{-} e_{u}^{0} \mathscr{P}^{-} e_{u}^{n} \mathrm{~d} x \\
& \quad-\alpha_{0} \int_{\Omega} r^{n}(x) \mathscr{P}^{-} e_{u}^{n} \mathrm{~d} x+\int_{\Omega}\left(\mathscr{P}^{-} u\left(x, t_{n}\right)-u\left(x, t_{n}\right)\right) \mathscr{P}^{-} e_{u}^{n} \mathrm{~d} x \\
& \quad+\alpha_{0}\left(\Delta_{(\beta-2) / 2}\left(\mathscr{Q} p\left(x, t_{n}\right)-p\left(x, t_{n}\right)\right)-\left(\mathscr{P}^{+} q\left(x, t_{n}\right)\right.\right. \\
& \left.\left.\quad-q\left(x, t_{n}\right)\right), \mathscr{Q} e_{p}^{n}\right) \\
& \quad-\alpha_{0} \sqrt{\varepsilon}\left(\mathscr{P}^{+} q\left(x, t_{n}\right)-q\left(x, t_{n}\right)^{-}\right)_{N+(1 / 2)}\left(\mathscr{P}^{-} e_{u}^{n}\right)_{N+(1 / 2)}^{-} \\
& \quad-\sum_{i=1}^{n-1}\left(b_{i-1}-b_{i}\right) \int_{\Omega}\left(\mathscr{P}^{-} u\left(x, t_{n-i}\right)-u\left(x, t_{n-i}\right)\right) \mathscr{P}^{-} e_{u}^{n} \mathrm{~d} x \\
& \quad-b_{n-1} \int_{\Omega}\left(\mathscr{P}^{-} u\left(x, t_{0}\right)-u\left(x, t_{0}\right)\right) \mathscr{P}^{-} e_{u}^{n} \mathrm{~d} x+\mathscr{F}\left(f: u, u_{h}: v\right) .
\end{aligned}
$$

Similarly, we analyze the error estimate by applying the mathematical induction method. First, we consider the case when $n=1$, equation (56) becomes

$$
\begin{aligned}
& \left\|\mathscr{P}^{-} e_{u}^{1}\right\|^{2}+\alpha_{0}\left(\Delta_{(\beta-2) / 2} Q e_{p}^{1}, \mathscr{Q} e_{p}^{1}\right)+\frac{\alpha_{0} \sqrt{\varepsilon} \mu}{h}\left(\mathscr{P}^{-} e_{u}^{1}\right)_{N+(1 / 2)}^{2} \\
& =\int_{\Omega} \mathscr{P}^{-} e_{u}^{0} \mathscr{P}^{-} e_{u}^{1} \mathrm{~d} x-\alpha_{0} \int_{\Omega} r^{1}(x) \mathscr{P}^{-} e_{u}^{1} \mathrm{~d} x \\
& \quad+\int_{\Omega}\left(\mathscr{P}^{-} u\left(x, t_{1}\right)-u\left(x, t_{1}\right)\right) \mathscr{P}^{-} e_{u}^{1} \mathrm{~d} x \\
& \quad+\alpha_{0}\left(\Delta_{(\beta-2) / 2}\left(Q \mathcal{Q} p\left(x, t_{1}\right)-p\left(x, t_{1}\right)\right)-\left(\mathscr{P}^{+} q\left(x, t_{1}\right)-q\left(x, t_{1}\right)\right), \mathscr{Q} e_{p}^{1}\right) \\
& \quad-\alpha_{0} \sqrt{\varepsilon}\left(\mathscr{P}^{+} q\left(x, t_{1}\right)-q\left(x, t_{1}\right)^{-}\right)_{N+(1 / 2)}\left(\mathscr{P}^{-} e_{u}^{1}\right)_{N+(1 / 2)}^{-} \\
& \quad-\int_{\Omega}\left(\mathscr{P}^{-} u\left(x, t_{0}\right)-u\left(x, t_{0}\right)\right) \mathscr{P}^{-} e_{u}^{1} \mathrm{~d} x+\mathscr{F}\left(f: u, u_{h}: v\right) .
\end{aligned}
$$

Notice that

$$
\begin{gathered}
\left\|\mathscr{P}^{-} e_{u}^{0}\right\| \leq C h^{k+1}, \\
a b \leq \varepsilon a^{2}+\frac{1}{4 \varepsilon} b^{2} .
\end{gathered}
$$

$$
\begin{aligned}
\left\|\Delta_{(\beta-2) / 2}\left(\mathscr{Q} p\left(x, t_{1}\right)-p\left(x, t_{1}\right)\right)-\left(\mathscr{P}^{+} q\left(x, t_{1}\right)-q\left(x, t_{1}\right)\right)\right\| \\
=\| \Delta_{(\beta-2) / 2}\left(\mathscr{Q} p\left(x, t_{1}\right)-p\left(x, t_{1}\right)\right)-\triangle_{(\beta-2) / 2}\left(\mathscr{P}^{+} p\left(x, t_{1}\right)\right. \\
\left.\quad-p\left(x, t_{1}\right)\right) \| \\
\quad \leq C h^{k+1} .
\end{aligned}
$$

Combining with the standard approximation theorem (17), we obtain 
Combining Lemmas 9 and 10 in [24] with Definition 2.4, equation (46), and Young's inequality, we obtain

$$
\begin{aligned}
\| \mathscr{P}^{-} & e_{u}^{1} \|^{2}+\alpha_{0}\left(\Delta_{(\beta-2) / 2} Q \mathcal{Q} e_{p}^{1}, \mathcal{Q} e_{p}^{1}\right)+\frac{\alpha_{0} \sqrt{\varepsilon} \mu}{h}\left(\mathscr{P}^{-} e_{u}^{1}\right)_{N+(1 / 2)}^{2} \\
\leq & \left(\left\|\mathscr{P}^{-} e_{u}^{0}\right\|+\alpha_{0}\left\|r^{1}(x)\right\|+\left\|\mathscr{P}^{-} u\left(x, t_{1}\right)-u\left(x, t_{1}\right)\right\|+\left\|\mathscr{P}^{-} u\left(x, t_{0}\right)-u\left(x, t_{0}\right)\right\|\right) \\
& \times\left\|\mathscr{P}^{-} e_{u}^{1}\right\|+C h^{2 k+2} \tau^{\alpha}+\frac{\alpha_{0}}{\varepsilon C}\left\|Q e_{p}^{1}\right\|^{2}+\frac{\alpha_{0} \sqrt{\varepsilon} \mu}{h}\left(\mathscr{P}^{-} e_{u}^{1}\right)_{N+(1 / 2)}^{2}-\frac{1}{4} a\left(\hat{f} ; u_{h}\right)[v]^{2} \tau^{\alpha} \\
& +\left(C+C_{*}\left(\|v\|_{\infty}+h^{-1}\left\|e_{u}\right\|_{\infty}^{2}\right)\right)\|v\|_{L^{2}(\Omega)}^{2} \tau^{\alpha}+\left(C+C_{*} h^{-1}\left\|e_{u}\right\|_{\infty}^{2}\right) h^{2 k+1} \tau^{\alpha} .
\end{aligned}
$$

Thus,

$$
\begin{aligned}
&\left\|\mathscr{P}^{-} e_{u}^{1}\right\|^{2}+\alpha_{0}\left(\Delta_{(\beta-2) / 2} Q e_{p}^{1}, Q e_{p}^{1}\right) \\
& \leq C\left(h^{k+1}+\tau^{2}\right)^{2}+\varepsilon\left\|\mathscr{P}^{-} e_{u}^{1}\right\|^{2}+C h^{2 k+2} \tau^{\alpha}+\frac{\alpha_{0}}{\varepsilon C}\left\|Q e_{p}^{1}\right\|^{2} \\
&-\frac{1}{4} a\left(\hat{f} ; u_{h}\right)[v]^{2} \tau^{\alpha} \\
&+\left(C+C_{*}\left(\|v\|_{\infty}+h^{-1}\left\|e_{u}\right\|_{\infty}^{2}\right)\right)\|v\|_{L^{2}(\Omega)}^{2} \tau^{\alpha} \\
&+\left(C+C_{*} h^{-1}\left\|e_{u}\right\|_{\infty}^{2}\right) h^{2 k+1} \tau^{\alpha} .
\end{aligned}
$$

If we choose $\varepsilon \leq \min \{1,1 / C\}$ and recall the fractional Poincaré-Friedrichs, Lemmas 7 and 9 and inequality (53), the standard approximation theorem (17), and the positive property of $a\left(\widehat{f} ; u_{h}\right)$, we obtain

$$
\left\|\mathscr{P}^{-} e_{u}^{1}\right\| \leq b_{0}^{-1} C\left(h^{k+1}+\tau^{2}+\tau^{\alpha / 2} h^{k+(1 / 2)}\right),
$$

where $C$ is a positive constant which depends on $u, T, \alpha$.

Next, we assume that the following inequality holds:

$$
\left\|\mathscr{P}^{-} e_{u}^{m}\right\| \leq b_{m-1}^{-1} C\left(h^{k+1}+\tau^{2}+\tau^{\alpha / 2} h^{k+(1 / 2)}\right), \quad m=1,2, \ldots, l .
$$

When $n=l+1$, from equation (56) and Young's inequality, we obtain

$$
\begin{aligned}
\| \mathscr{P}^{-} & e_{u}^{l+1} \|^{2}+\alpha_{0}\left(\Delta_{(\beta-2) / 2} \mathscr{Q} e_{p}^{l+1}, Q_{e}^{l+1}\right)+\frac{\alpha_{0} \sqrt{\varepsilon} \mu}{h}\left(\mathscr{P}^{-} e_{u}^{l+1}\right)_{N+(1 / 2)}^{2} \\
= & \sum_{i=1}^{l}\left(b_{i-1}-b_{i}\right) \int_{\Omega} \mathscr{P}^{-} e_{u}^{l+1-i} \mathscr{P}^{-} e_{u}^{l+1} \mathrm{~d} x+b_{l} \int_{\Omega} \mathscr{P}^{-} e_{u}^{0} \mathscr{P}^{-} e_{u}^{l+1} \mathrm{~d} x \\
& -\alpha_{0} \int_{\Omega} r^{l+1}(x) \mathscr{P}^{-} e_{u}^{l+1} \mathrm{~d} x+\int_{\Omega}\left(\mathscr{P}^{-} u\left(x, t_{l+1}\right)-u\left(x, t_{l+1}\right)\right) \mathscr{P}^{-} e_{u}^{l+1} \mathrm{~d} x \\
& +\alpha_{0}\left(\Delta_{(\beta-2) / 2}\left(\mathscr{Q} p\left(x, t_{l+1}\right)-p\left(x, t_{l+1}\right)\right)-\left(\mathscr{P}^{+} q\left(x, t_{l+1}\right)-q\left(x, t_{l+1}\right)\right), \mathscr{Q} e_{p}^{l+1}\right) \\
& -\alpha_{0} \sqrt{\varepsilon}\left(\mathscr{P}^{+} q\left(x, t_{l+1}\right)-q\left(x, t_{l+1}\right)^{-}\right)_{N+(1 / 2)}\left(\mathscr{P}^{-} e_{u}^{l+1}\right)_{N+(1 / 2)}^{-} \\
& -\sum_{i=1}^{l}\left(b_{i-1}-b_{i}\right) \int_{\Omega}\left(\mathscr{P}^{-} u\left(x, t_{l+1-i}\right)-u\left(x, t_{l+1-i}\right)\right) \mathscr{P}^{-} e_{u}^{l+1} \mathrm{~d} x \\
& -b_{l} \int_{\Omega}\left(\mathscr{P}^{-} u\left(x, t_{0}\right)-u\left(x, t_{0}\right)\right) \mathscr{P}^{-} e_{u}^{l+1} \mathrm{~d} x+\mathscr{F}^{\prime}\left(f: u, u_{h}: v\right) . \\
\leq & \left(\sum_{i=1}^{l}\left(b_{i-1}-b_{i}\right)\left\|\mathscr{P}^{-} e_{u}^{l+1-i}\right\|+b_{l}\left\|\mathscr{P}^{-} e_{u}^{0}\right\|+\alpha_{0}\left\|r^{l+1}(x)\right\|+\left\|\mathscr{P}^{-} u\left(x, t_{l+1}\right)-u\left(x, t_{l+1}\right)\right\|\right. \\
& +\sum_{i=1}^{l}\left(b_{i-1}-b_{i}\right)\left\|\mathscr{P}^{-} u\left(x, t_{l+1-i}\right)-u\left(x, t_{l+1-i}\right)\right\|+b_{l}\left\|\mathscr{P}^{-} u\left(x, t_{0}\right)-u\left(x, t_{0}\right)\right\| \\
& \times\left\|\mathscr{P}^{-} e_{u}^{l+1}\right\|+C h^{2 k+2} \tau^{\alpha}+\frac{\alpha_{0}}{\varepsilon C}\left\|\mathscr{Q} e_{p}^{l+1}\right\|^{2}+\frac{\alpha_{0} \sqrt{\varepsilon} \mu}{h}\left(\mathscr{P}^{-} e_{u}^{l+1}\right)_{N+(1 / 2)}^{2}-\frac{1}{4} a\left(\hat{f} ; u_{h}\right)[v]^{2} \tau^{\alpha} \\
& +\left(C+C_{*}\left(\|v\|_{\infty}+h^{-1}\left\|e_{u}\right\|_{\infty}^{2}\right)\right)\|v\|_{L^{2}(\Omega)}^{2} \tau^{\alpha}+\left(C+C_{*} h^{-1}\left\|e_{u}\right\|_{\infty}^{2}\right) h^{2 k+1} \tau^{\alpha} .
\end{aligned}
$$


Because

$$
b_{i-1}^{-1}<b_{i}^{-1}, \tau^{\alpha / 2} h^{k+1}>0
$$

$$
\begin{aligned}
\| \mathscr{P}^{-} & e_{u}^{l+1} \|^{2}+\alpha_{0}\left(\Delta_{(\beta-2) / 2} Q e_{p}^{l+1}, Q e_{p}^{l+1}\right)+\frac{\alpha_{0} \sqrt{\varepsilon} \mu}{h}\left(\mathscr{P}^{-} e_{u}^{l+1}\right)_{N+(1 / 2)}^{2} \\
\leq & \left(\sum_{i=1}^{l}\left(b_{i-1}-b_{i}\right) b_{l}^{-1} C\left(h^{k+1}+\tau^{2}+\tau^{\alpha / 2} h^{k+1}\right)+b_{l} b_{l}^{-1} C\left(h^{k+1}+\tau^{2}+\tau^{\alpha / 2} h^{k+1}\right)\right) \\
& \times\left\|\mathscr{P}^{-} e_{u}^{l+1}\right\|+C h^{2 k+2} \tau^{\alpha}+\frac{\alpha_{0}}{\varepsilon C}\left\|\mathbb{Q}_{p}^{l+1}\right\|^{2}+\frac{\alpha_{0} \sqrt{\varepsilon} \mu}{h}\left(\mathscr{P}^{-} e_{u}^{l+1}\right)_{N+(1 / 2)}^{2} \\
& -\frac{1}{4} a\left(\widehat{f} ; u_{h}\right)[v]^{2} \tau^{\alpha}+\left(C+C_{*}\left(\|v\|_{\infty}+h^{-1}\left\|e_{u}\right\|_{\infty}^{2}\right)\right)\|v\|_{L^{2}(\Omega)}^{2} \tau^{\alpha}+\left(C+C_{*} h^{-1}\left\|e_{u}\right\|_{\infty}^{2}\right) h^{2 k+1} \tau^{\alpha} \\
\leq & \left(\left(\sum_{i=1}^{l}\left(b_{i-1}-b_{i}\right)+b_{l}\right) b_{l}^{-1} C\left(h^{k+1}+\tau^{2}+\tau^{\alpha / 2} h^{k+1}\right)\right) \times\left\|\mathscr{P}^{-} e_{u}^{l+1}\right\| \\
& +C h^{2 k+2} \tau^{\alpha}+\frac{\alpha_{0}}{\varepsilon C}\left\|{ }_{Q} e_{p}^{l+1}\right\|^{2}+\frac{\alpha_{0} \sqrt{\varepsilon} \mu}{h}\left(\mathscr{P}^{-} e_{u}^{l+1}\right)_{N+(1 / 2)}^{2}-\frac{1}{4} a\left(\widehat{f} ; u_{h}\right)[v]^{2} \tau^{\alpha} \\
& +\left(C+C_{*}\left(\|v\|_{\infty}+h^{-1}\left\|e_{u}\right\|_{\infty}^{2}\right)\right)\|v\|_{L^{2}(\Omega)}^{2} \tau^{\alpha}+\left(C+C_{*} h^{-1}\left\|e_{u}\right\|_{\infty}^{2}\right) h^{2 k+1} \tau^{\alpha} .
\end{aligned}
$$

Analogously, apply Young's inequality to equation(66) and choose sufficiently small $\epsilon$; by using the fractional Poincaré-Friedrichs, Lemmas 7 and 9 and inequality (53), the standard approximation theorem (17), and the positive property of $a\left(\hat{f} ; u_{h}\right)$ again, we obtain

$$
\left\|\mathscr{P}^{-} e_{u}^{l+1}\right\| \leq b_{l}^{-1} C\left(h^{k+1}+\tau^{2}+\tau^{\alpha / 2} h^{k+(1 / 2)}\right),
$$

where $C$ is a positive constant which depends on $u, T, \alpha$.

According to the mathematical induction method, we get

$$
\mathscr{P}^{-} e_{u}^{n} \leq b_{n-1}^{-1} C\left(h^{k+1}+\tau^{2}+\tau^{\alpha / 2} h^{k+(1 / 2)}\right)
$$

From [39], we know that the term $n^{-\alpha} b_{n-1}^{-1}$ monotonic increase tends to $(1 /(1-\alpha))(n \longrightarrow \infty)$; then,

$$
\begin{aligned}
& \left\|\mathscr{P}^{-} e_{u}^{n}\right\| \leq b_{n-1}^{-1} C\left(h^{k+1}+\tau^{2}+\tau^{\alpha / 2} h^{k+(1 / 2)}\right) \\
& \leq n^{\alpha} n^{-\alpha} b_{n-1}^{-1} C\left(h^{k+1}+\tau^{2}+\tau^{\alpha / 2} h^{k+(1 / 2)}\right) \\
& \leq\left(\frac{T}{\tau}\right)^{\alpha} \frac{1}{1-\alpha} C\left(h^{k+1}+\tau^{2}+\tau^{\alpha / 2} h^{k+(1 / 2)}\right) \\
& \leq \frac{C_{u} T^{\alpha}}{1-\alpha}\left(\tau^{-\alpha} h^{k+1}+\tau^{2-\alpha}+\tau^{-\alpha / 2} h^{k+(1 / 2)}\right),
\end{aligned}
$$

where $C_{u}$ is a constant which only depends on $u$.

When $\alpha \longrightarrow 1,1 /(1-\alpha) \longrightarrow \infty$, estimation formula (69) is meaningless, so we should re-estimate the case for $\alpha \longrightarrow 1$.
Again, we can prove the following estimator by using mathematical induction:

$$
\left\|\mathscr{P}^{-} e_{u}^{n}\right\| \leq n C\left(h^{k+1}+\tau^{2}+\tau^{\alpha / 2} h^{k+(1 / 2)}\right) .
$$

The proof techniques are almost the same as the subdiffusion case in [24], and in order to save space, here, we ignore the concrete proof; thus, when $\alpha \longrightarrow 1$, we obtain

$$
\begin{gathered}
\left\|\mathscr{P}^{-} e_{u}^{n}\right\| \leq n C\left(h^{k+1}+\tau^{2}+\tau^{\alpha / 2} h^{k+(1 / 2)}\right) \\
\leq T C_{u}\left(\tau^{-1} h^{k+1}+\tau+\tau^{-1 / 2} h^{k+(1 / 2)}\right),
\end{gathered}
$$

where $C_{u}$ is a constant which only depends on $u$.

According to inequalities (69) and (71), the triangle inequality, and the standard approximation theorem (17), coupling with the property of the projection operator, we can prove that the results of Theorem 3 hold.

Theorem 4. Let $u\left(x, t_{n}\right)$ be the exact solution of nonlinear superdiffusion problem (2), which is sufficiently smooth with bounded derivatives, and assume that $f \in C^{3}$, let $u_{h}^{n}$ denote the numerical solution of the LDG scheme (28), and use $e_{u}=$ $u\left(x, t_{n}\right)-u_{h}^{n}$ to denote the corresponding numerical error; then, the following error estimates hold:

When $1<\alpha<2$,

$$
\left\|u\left(x, t_{n}\right)-u_{h}^{n}\right\| \leq \frac{C_{u} T^{\alpha-1}}{2-\alpha}\left(\tau^{1-\alpha} h^{k+1}+\tau^{3-\alpha}+\tau^{1-\alpha / 2} h^{k+1 / 2}+h^{k+1}\right),
$$

and when $\alpha \longrightarrow 2$, 


$$
\left\|u\left(x, t_{n}\right)-u_{h}^{n}\right\| \leq C_{u} T\left(\tau^{-1} h^{k+1}+\tau+h^{k+1 / 2}+h^{k+1}\right),
$$

where $C_{u}$ is a constant which only depends on $u$.
Proof. Subtracting equation (28) from equation (27) and combining with the fluxes on boundaries, we obtain the following error equation:

$$
\begin{aligned}
& \int_{\Omega} e_{u}^{n} v \mathrm{~d} x+\alpha_{1} \sqrt{\varepsilon}\left(\int_{\Omega} e_{q}^{n} v_{x} \mathrm{~d} x-\sum_{j=1}^{N}\left(\left(e_{q}^{n}\right)^{+} v_{j+(1 / 2)}^{-}-\left(e_{q}^{n}\right)^{+} v_{j-(1 / 2)}^{+}\right)\right) \\
& -\alpha_{1} \int_{\Omega}\left(f\left(u\left(x, t_{n}\right)\right)-f\left(u_{h}^{n}\right)\right) v_{x} \mathrm{~d} x+\alpha_{1} \sum_{j=1}^{N}\left(f\left(u\left(x, t_{n}\right)\right)-\widehat{f}\left(u_{h}^{n}\right)\right) v_{j+(1 / 2)}^{-} \\
& -\alpha_{1} \sum_{j=1}^{N}\left(f\left(u\left(x, t_{n}\right)\right)-\widehat{f}\left(u_{h}^{n}\right)\right) v_{j-(1 / 2)}^{+}-\sum_{i=1}^{n-2}\left(-v_{i-1}+2 v_{i}-v_{i+1}\right) \int_{\Omega} e_{u}^{n-i-1} v \mathrm{~d} x \\
& -\left(2 v_{0}-v_{1}\right) \int_{\Omega} e_{u}^{n-1} v \mathrm{~d} x-\left(2 v_{n-1}-v_{n-2}\right) \int_{\Omega} e_{u}^{0} v \mathrm{~d} x+v_{n-1} \int_{\Omega} e_{u}^{-1} v \mathrm{~d} x \\
& +\alpha_{1} \int_{\Omega} R^{n}(x) v \mathrm{~d} x+\int_{\Omega} e_{q}^{n} w \mathrm{~d} x-\int_{\Omega} \Delta_{(\beta-2) / 2} e_{p}^{n} w \mathrm{~d} x+\int_{\Omega} e_{p}^{n} z \mathrm{~d} x \\
& +\sqrt{\varepsilon}\left(\int_{\Omega} e_{u}^{n} z_{x} \mathrm{~d} x-\sum_{j=1}^{N}\left(\left(e_{u}^{n}\right)^{-} z_{j+(1 / 2)}^{-}-\left(e_{u}^{n}\right)^{-} z_{j-(1 / 2)}^{+}\right)\right)_{n} 0
\end{aligned}
$$

Similar to the error estimate of the nonlinear subdiffusion case, together with the following error estimator

$$
\left\|e_{u}^{-1}\right\| \leq C\left(h^{k+1}+\tau^{2}+\tau h^{k+1}\right)
$$

where $C$ is a constant related to $u, T, \alpha$ [24], we can prove that the following error inequality holds by using mathematical induction:

$$
\left\|\mathscr{P}^{-} e_{u}^{n}\right\| \leq v_{n-1}^{-1} C\left(h^{k+1}+\tau^{2}+\tau^{\alpha / 2} h^{k+1 / 2}\right) .
$$

According to Qiu et al. [24], we know that $n^{1-\alpha} v_{n-1}^{-1}$ monotone increasing tends to $1 /(2-\alpha)(n \longrightarrow \infty)$, so

$$
\begin{aligned}
\left\|\mathscr{P}^{-} e_{u}^{n}\right\| & \leq v_{n-1}^{-1} C\left(h^{k+1}+\tau^{2}+\tau^{\alpha / 2} h^{k+1 / 2}\right) \\
& \leq n^{\alpha-1} n^{1-\alpha} \nu_{n-1}^{-1} C\left(h^{k+1}+\tau^{2}+\tau^{\alpha / 2} h^{k+1 / 2}\right) \\
& \leq\left(\frac{T}{\tau}\right)^{\alpha-1} \frac{1}{2-\alpha} C\left(h^{k+1}+\tau^{2}+\tau^{\alpha / 2} h^{k+1 / 2}\right) \\
& \leq \frac{C_{u} T^{\alpha-1}}{2-\alpha}\left(\tau^{1-\alpha} h^{k+1}+\tau^{3-\alpha}+(\tau)^{1-(\alpha / 2)} h^{k+1 / 2}\right),
\end{aligned}
$$

where $C_{u}$ is a constant which only depends on $u$.

When $\alpha \longrightarrow 2$ and $1 /(2-\alpha) \longrightarrow \infty$, the estimator (77) is insignificant; hence, we should re-estimate the case for $\alpha \longrightarrow 2$.

Similar to the nonlinear subdiffusion case, we still use the mathematical induction to prove the following estimator:

$$
\left\|\mathscr{P}^{-} e_{u}^{n}\right\| \leq n C\left(h^{k+1}+\tau^{2}+\tau^{\alpha / 2} h^{k+1 / 2}\right) .
$$

The proof techniques are almost the same as the superdiffusion case in [24]; for simplicity, here, we ignore the proof process; thus, when $\alpha \longrightarrow 2$, we obtain

$$
\begin{aligned}
\mathscr{P}^{-} e_{u}^{n} & \leq n C\left(h^{k+1}+\tau^{2}+\tau^{\alpha / 2} h^{k+1 / 2}\right) \\
& \leq T C_{u}\left(\tau^{-1} h^{k+1}+\tau+h^{k+1 / 2}\right),
\end{aligned}
$$

where $C_{u}$ is a constant which only depends on $u$.

According to inequalities (77) and (79), the triangle inequality, and the standard approximation theorem (17), coupling with the property of the projection operator, we can prove that the results of Theorem 4 hold.

\section{Implementation Procedure}

In this part, we give out several examples to demonstrate the effectiveness of the finite difference/LDG approximation for solving nonlinear time-space fractional subdiffusion/ superdiffusion equations. At the same time, we propose the convergence behavior of numerical solutions with respect to the time step size $\tau$ and/or the space step size $h$, i.e., through carefully choosing the appropriate time step size, and let the Lax-Friedrichs numerical flux be a numerical flux function of the nonlinear terms $\widehat{f}$; we observe that a convergence rate can arrive at $\mathcal{O}\left(\tau^{2-\alpha}+h^{k+1}\right)$ when $0<\alpha<1$ and approach to $\mathcal{O}\left(\tau^{3-\alpha}+h^{k+1}\right)$ when the derivative parameter is $1<\alpha<2$.

6.1. Numerical Examples. As examples, we consider the following nonlinear time-space fractional subdiffusion/ superdiffusion equations: 


$$
\begin{cases}\frac{\partial^{\alpha} u(x, t)}{\partial t^{\alpha}}+u(x, t) \frac{\partial u(x, t)}{\partial x}-a(x) \varepsilon\left(-(-\Delta)^{\beta / 2}\right) u(x, t)=h(x, t), & x \in \Omega, t \in[0, T], \\ u(x, 0)=u_{0}(x), & x \in \Omega, \\ u(x, t)=0, & x \in \partial \Omega, t \in[0, T],\end{cases}
$$

where $\Omega=[a, b], f(u)=1 / 2 u^{2}$, and $T>0$; when $0<\alpha<1$, it is the nonlinear subdiffusion case, and when the derivative parameter is $1<\alpha<2$, it is the nonlinear superdiffusion case, and $1<\beta<2, \epsilon>0$.

For brevity, we only derive the linear system obtained from the fully discrete LDG scheme (24). Similarly, we can get the corresponding linear system of scheme (28).
We consider the case of discontinuous piecewise polynomial basis function sequences $\left\{\phi_{i}(x)\right\}_{i=1}^{k}$ in the space $V_{k}^{h}$. By the definition of the space $V_{k}^{h}$, we know that, for all $v \in V_{k}^{h}$, there holds $v(x)=\sum_{i=1}^{N} v_{i} \phi_{i}(x)$, where $v_{i}=v\left(x_{i}\right)$.

From the LDG scheme (24), we obtain the fully discrete scheme of equation (80) as follows:

$$
\begin{aligned}
& \int_{\Omega} u_{h}^{n} v \mathrm{~d} x-\alpha_{0} \int_{\Omega} f\left(u_{h}^{n}\right) v_{x} \mathrm{~d} x+\alpha_{0} \sum_{j=1}^{N}\left(\widehat{f}\left(u_{h}^{n}\right) v_{j+(1 / 2)}^{-}-\widehat{f}\left(u_{h}^{n}\right) v_{j-(1 / 2)}^{+}\right) \\
& +\alpha_{0} \sqrt{\varepsilon} a(x)\left(\int_{\Omega} q_{h}^{n} v_{x} \mathrm{~d} x-\sum_{j=1}^{N}\left(\widehat{q_{h}^{n}} v_{j+(1 / 2)}^{-}-\widehat{q_{h}^{n}} v_{j-(1 / 2)}^{+}\right)\right) \\
& =\sum_{i=1}^{n-1}\left(b_{i-1}-b_{i}\right) \int_{\Omega} u_{h}^{n-i} v \mathrm{~d} x+b_{n-1} \int_{\Omega} u_{h}^{0} v \mathrm{~d} x+\int_{\Omega} h(x, t) v \mathrm{~d} x \\
& \int_{\Omega} q_{h}^{n} w \mathrm{~d} x-\int_{\Omega} \Delta_{(\beta-2) / 2} p_{h}^{n} w \mathrm{~d} x=0, \\
& \int_{\Omega} p_{h}^{n} z \mathrm{~d} x+\sqrt{\varepsilon} a(x)\left(\int_{\Omega} u_{h}^{n} z_{x} \mathrm{~d} x-\sum_{j=1}^{N}\left(\widehat{u_{h}^{n}} z_{j+(1 / 2)}^{-}-\widehat{u_{h}^{n}} z_{j-(1 / 2)}^{+}\right)\right)=0 \\
& \int_{\Omega} u_{h}^{0} v \mathrm{~d} x-\int_{\Omega} u_{0}(x) v \mathrm{~d} x=0,
\end{aligned}
$$

where $\alpha_{0}=\tau^{\alpha} \Gamma(2-\alpha)$.

In terms of the basis $\left\{\phi_{i}(x)\right\}_{i=1}^{N}$, we denote approximate solutions by $u_{h}^{n}=\sum_{i=1}^{N} u_{j}(t) \phi_{j}(x), p_{h}^{n}=\sum_{i=1}^{N} p_{j}(t) \phi_{j}(x)$, and $q_{h}^{n}=\sum_{i=1}^{N} q_{j}(t) \phi_{j}(x)$, and take test functions as $v=\sum_{i=1}^{N} v_{i} \phi_{i}(x), v_{i}=v\left(x_{i}\right), \quad w=\sum_{i=1}^{N} w_{i} \phi_{i}(x), w_{i}=w\left(x_{i}\right)$, and $z=\sum_{i=1}^{N} z_{i} \phi_{i}(x), z_{i}=z\left(x_{i}\right)$. Plug these expressions in the LDG scheme (81) and take flux equation (25); we get the linear system of the scheme in the $j$ th element at $t_{n}$ as follows:

$$
\begin{aligned}
& \left(\begin{array}{ccc}
M+\alpha_{0} B & 0 & \alpha_{0} \sqrt{\varepsilon}\left(A-H_{11}+H_{12}\right) \\
0 & R & M \\
\sqrt{\varepsilon}\left(A-H_{13}+H_{14}\right) & M & 0
\end{array}\right)\left(\begin{array}{c}
U_{j}^{n} \\
P_{j}^{n} \\
Q_{j}^{n}
\end{array}\right) \\
& =F_{n} v+\sum_{i=1}^{n-1}\left(b_{i-1}-b_{i}\right) U_{n-i} v+b_{n-1} U_{0} v
\end{aligned}
$$

where $B$ represents the combined part of nonlinear terms; here, we select the Lax-Friedrichs flux as a numerical flux function of nonlinear term $\widehat{f}\left(u_{h}^{n}\right), \quad M=\left\{\left(\phi_{m}^{j}(x)\right.\right.$, $\left.\left.\phi_{k}^{j}(x)\right)\right\}_{j=1, \ldots, N}$ is the mass matrix, $m, k=0,1, \ldots$, denote the polynomials order, and

$$
\begin{aligned}
& \mathrm{A}=\left\{\left(\phi_{m}^{j}(x), \phi_{k}^{\prime j}(x)\right)\right\}_{j=1, \ldots, N}, \\
& H_{11}=\left\{\left(\phi_{m}^{j}\left(x_{j-1 / 2}\right), \phi_{k}^{j}\left(x_{j+1 / 2}\right)\right)\right\}_{j=1, \ldots, N^{\prime}} \\
& H_{12}=\left\{\left(\phi_{m}^{j}\left(x_{j-1 / 2}\right), \phi_{k}^{j}\left(x_{j-1 / 2}\right)\right)\right\}_{j=1, \ldots, N^{\prime}} \\
& H_{13}=\left\{\left(\phi_{m}^{j}\left(x_{j+1 / 2}\right), \phi_{k}^{j}\left(x_{j+1 / 2}\right)\right)\right\}_{j=1, \ldots, N^{\prime}} \\
& H_{14}=\left\{\left(\phi_{m}^{j-1}\left(x_{j-1 / 2}\right), \phi_{k}^{j}\left(x_{j-1 / 2}\right)\right)\right\}_{j=1, \ldots, N^{*}}
\end{aligned}
$$

$R$ is a quadrature for fractional integral operator $\triangle_{(\beta-2) / 2} p_{h}^{n}$ and test function $w$ in the $j$ th element. Recalling the works in [34], we know that

$$
\Delta_{(\beta-2) / 2} p_{h}^{n}=-\frac{I_{x}^{2-\beta} p_{h}^{n}+{ }_{x} I_{b}^{2-\beta} p_{h}^{n}}{2 \cos (\beta \pi / 2)},
$$

where 


$$
\begin{aligned}
& { }_{a} I_{x}^{2-\beta} p_{h}^{n}(x, t)=\frac{1}{\Gamma(2-\beta)} \int_{a}^{x}(x-\xi)^{1-\beta} p_{h}^{n}(\xi, t) d_{\xi}, \\
& { }_{x} I_{b}^{2-\beta} p_{h}^{n}(x, t)=\frac{1}{\Gamma(2-\beta)} \int_{x}^{b}(\xi-x)^{1-\beta} p_{h}^{n}(\xi, t) d_{\xi},
\end{aligned}
$$

and $F_{n}=\left(h_{1}, h_{2}, \ldots, h_{n}\right)^{T}$ is a vector-valued function, $h_{j}\left(t_{n}\right)=h\left(x_{j}, t_{n}\right)$. Then, by solving linear system (82), we can solve the solution $U_{n}$. This study uses MATLAB programs to execute the mathematical calculations involved in the proposed numerical method.

\subsection{Numerical Results}

Example 1. Consider the nonlinear fractional time-space subdiffusion $(0<\alpha<1)$ equation (80), and satisfy initial boundary conditions:

$$
\begin{aligned}
& u(x, 0)=x^{2}(1-x), \quad x \in[0,1], \\
& u(0, t)=u(1, t)=0, \quad t>0 .
\end{aligned}
$$

In order to validate the optimal convergence of the proposed method, we select $a(x)=\Gamma(2.8) x / 2, \varepsilon=1$ and the sourcelterm as

$$
h(x, t)=\frac{2 x^{2}(1-x) t^{1.2}}{\Gamma(2.2)+0.2 x^{1.8}\left(1+t^{2}\right)} .
$$

Thus, equation (80) has an exact solution $u(x, t)=x^{2}(1-x)\left(1+t^{2}\right)$.

By choosing the fluxes in equation (25) for the linear term and Lax-Friedrichs flux for the nonlinear term $\widehat{f}$ and adopting the linear piecewise polynomials, then we numerically solve equation (80). We investigate the $L^{2}$ errors and the corresponding convergence rate of the solutions with respect to different fractional-order parameters $\alpha$ and $\beta$. First, we select the appropriate time step size $\tau$ such that the time discretization errors are negligible as compared with the spatial errors. Then, choose the space step size sequence as $h=1 / 2^{i},(i=2, \ldots, 6)$; Tables 1 and 2 list out the $L^{2}$ errors and the corresponding convergence order, respectively, which is fixed the value of one parameter $\beta$ (or $\alpha$ ), with the values change of the other parameter $\alpha(\operatorname{or} \beta)$. As can be seen from the data in the two tables, our schemes can achieve the optimal convergence $\mathcal{O}\left(h^{k+1}\right)$ in spatial direction; this matches the theoretical results of Theorem 3.

The numerical data listed in Tables 3 and 4 show that when we choose the appropriate space step size $h$ and the time step size sequence $\tau=1 / 2^{i},(i=2, \ldots, 6)$, fix the values of one derivative parameter $\beta$ (or $\alpha$ ), and then change the values of the other derivative constant $\alpha$ (or $\beta$ ), when $0<\alpha<1$, the accuracy in time direction of the nonlinear time-space fractional subdiffusion equation can achieve to $\mathcal{O}\left(\tau^{2-\alpha}\right)$ under the $L^{2}$ norm and can reach to 1 when $\alpha \longrightarrow 1$; this experiment result agrees with the theoretical computation results in Theorem 3 .

Although an order of convergence $\mathcal{O}\left(h^{k+1}\right)$ is predicted by Theorem 3 in the spatial direction for any monotone flux of the nonlinear term, however, optimal order of superconvergence is observed when we choose a Lax-Friedrichs flux for the nonlinear term $\widehat{f}$ and an alternating direction flux for the linear term. We do not understand the reason for this improved rate at present, but it may be associated with the "global dependency" of the fractional operator which exhibits optimal convergence.

Example 2. Consider the following nonlinear time-space fractional subdiffusion $(0<\alpha<1)$ equation with Dirichlet boundary conditions:

$$
\begin{cases}\frac{\partial^{\alpha} u(x, t)}{\partial t^{\alpha}}+u(x, t) \frac{\partial u(x, t)}{\partial x}+c(x) u(x, t) \varepsilon\left(-(-\triangle)^{\frac{\beta}{2}}\right) u(x, t), & \text { in } x \in \Omega, t \in[0, T], \\ u(x, 0)=0, & \text { in } x \in \Omega, \\ u(-1, t)=u(1, t)=0, & \text { in } x \in \partial \Omega, t \in[0, T] .\end{cases}
$$

Here, $\Omega=[-1,1], T=1, c(x)=x^{2}+1, \quad \epsilon=0.5$; the equation has an exact solution $u(x, t)=\sin (t) C_{x+1}(2,2 \pi)$. Here,

$$
C_{x+n}(\gamma, \omega) \triangleq(x+n)^{\gamma} \sum_{j=0}^{\infty} \frac{(-1)^{j}(\omega(x+n))^{2 j}}{\Gamma(\gamma+2 j+1)} .
$$

In order to complete the scheme, we choose an alternating direction flux for the linear term and Lax-Friedrichs flux for the nonlinear term. Tables 5 and 6 reveal the $L^{2}$ norm and the convergence rate, respectively, in which one of the parameters $\beta$ (or $\alpha$ ) is fixed and the other parameter $\alpha$ (or $\beta$ ) is changed, with an appropriate time step size $\tau$.
Analogously, if we choose an appropriate space step size $h$ and fix one parameter $\beta$ (or $\alpha$ ) and then change the other constant $\alpha$ (or $\beta$ ), the results in Tables 7 and 8 show that when $0<\alpha<1$, the convergence rate in time direction of the nonlinear time-space fractional subdiffusion equation can converge to $\mathcal{O}\left(\tau^{2-\alpha}\right)$ under the $L^{2}$ norm, and the rate can be close to 1 when $\alpha \longrightarrow 1$; this result agrees with the theoretical analysis conclusion in Theorem 3 .

Example 3. In this example, we consider the nonlinear timespace fractional superdiffusion case $1<\alpha<2$ with the nonhomogeneous boundary condition: 
TABLE 1: The spatial convergence rate can achieve to $\mathcal{O}\left(h^{k+1}\right)$; here, $\beta=1.6$ and $T=1$.

\begin{tabular}{lccccc}
\hline$h$ & $\alpha=0.1$ & Rate & $\alpha=0.6$ & Rate & $\alpha=0.99$ \\
\hline $1 / 4$ & $7.1268 \times 10^{-2}$ & - & $6.3749 \times 10^{-2}$ & - & $6.7888 \times 10^{-2}$ \\
$1 / 8$ & $1.9575 \times 10^{-2}$ & 1.8642 & $1.6376 \times 10^{-2}$ & 1.9608 & $1.8660 \times 10^{-2}$ \\
$1 / 16$ & $5.2918 \times 10^{-3}$ & 1.8872 & $4.1156 \times 10^{-3}$ & 1.9924 & $4.9554 \times 10^{-3}$ \\
$1 / 32$ & $1.3757 \times 10^{-3}$ & 1.9436 & $1.0279 \times 10^{-3}$ & 2.0013 & $1.2498 \times 10^{-3}$ \\
$1 / 64$ & $3.4718 \times 10^{-4}$ & 1.9864 & $2.5439 \times 10^{-4}$ & 2.0147 & $3.1373 \times 10^{-4}$ \\
\hline
\end{tabular}

TABle 2: The spatial convergence rate can achieve to $\mathcal{O}\left(h^{k+1}\right)$; here, $\alpha=0.3$ and $T=1$.

\begin{tabular}{lccccc}
\hline$h$ & $\beta=1.01$ & Rate & $\beta=1.48$ & Rate & $\beta=1.97$ \\
\hline $1 / 4$ & $5.6314 \times 10^{-2}$ & - & $6.3326 \times 10^{-2}$ & - & $4.7829 \times 10^{-2}$ \\
$1 / 8$ & $1.4588 \times 10^{-2}$ & 1.9487 & $1.6475 \times 10^{-2}$ & 1.9425 & $1.3025 \times 10^{-2}$ \\
$1 / 16$ & $3.7356 \times 10^{-3}$ & 1.9615 & $4.2249 \times 10^{-3}$ & 1.9633 & $3.2786 \times 10^{-3}$ \\
$1 / 32$ & $9.4929 \times 10^{-4}$ & 1.9803 & $1.0590 \times 10^{-3}$ & 1.9962 & $8.2262 \times 10^{-4}$ \\
$1 / 64$ & $2.3761 \times 10^{-4}$ & 1.9982 & $2.6413 \times 10^{-4}$ & 2.0034 & 1.9901 \\
\hline
\end{tabular}

TABLE 3: The temporal convergence rate can arrive at $\mathcal{O}\left(\tau^{2-\alpha}\right) ; \beta=1.1$ and $T=1$.

\begin{tabular}{lccccc}
\hline$\tau$ & $\alpha=0.03$ & Rate & $\alpha=0.7$ & Rate & $\alpha=0.99$ \\
\hline $1 / 4$ & $6.0622 \times 10^{-3}$ & - & $6.3740 \times 10^{-3}$ & - & $4.0562 \times 10^{-3}$ \\
$1 / 8$ & $1.7016 \times 10^{-3}$ & 1.8329 & $2.7488 \times 10^{-3}$ & 1.2134 & $2.2067 \times 10^{-3}$ \\
$1 / 16$ & $4.5762 \times 10^{-4}$ & 1.8947 & $1.1518 \times 10^{-3}$ & 1.2549 & 0.8782 \\
$1 / 32$ & $1.1936 \times 10^{-4}$ & 1.9388 & $4.6859 \times 10^{-4}$ & 1.2975 & $6.1069 \times 10^{-4}$ \\
$1 / 64$ & $3.0484 \times 10^{-5}$ & 1.9692 & $1.9025 \times 10^{-4}$ & 1.3004 & $3.0668 \times 10^{-4}$ \\
\hline
\end{tabular}

TABLE 4: The temporal convergence rate can arrive at $\mathcal{O}\left(\tau^{2-\alpha}\right) ; \alpha=0.9$ and $T=1$.

\begin{tabular}{lccccc}
\hline$\tau$ & $\beta=1.1$ & Rate & $\beta=1.6$ & Rate & $\beta=1.98$ \\
\hline $1 / 4$ & $5.3762 \times 10^{-2}$ & - & $4.1429 \times 10^{-2}$ & - & $3.2679 \times 10^{-2}$ \\
$1 / 8$ & $2.6873 \times 10^{-2}$ & 1.0004 & $2.0677 \times 10^{-2}$ & 1.0026 & $1.6281 \times 10^{-2}$ \\
$1 / 16$ & $1.3400 \times 10^{-2}$ & 1.0029 & $1.0239 \times 10^{-2}$ & 1.0139 & $8.0914 \times 10^{-3}$ \\
$1 / 32$ & $6.3922 \times 10^{-3}$ & 1.0679 & $4.8191 \times 10^{-3}$ & 1.0873 & $3.9726 \times 10^{-3}$ \\
$1 / 64$ & $2.9818 \times 10^{-3}$ & 1.1001 & $2.2427 \times 10^{-3}$ & 1.1035 & 1.0051 \\
\hline
\end{tabular}

TABLE 5: The spatial convergence rate can achieve to $\mathcal{O}\left(h^{k+1}\right) ; \beta=1.6$ and $T=1$.

\begin{tabular}{lccccc}
\hline$h$ & $\alpha=0.1$ & Rate & $\alpha=0.5$ & Rate & $\alpha=0.9$ \\
\hline $1 / 4$ & $1.7936 \times 10^{-2}$ & - & $3.4408 \times 10^{-2}$ & - & $5.3221 \times 10^{-2}$ \\
$1 / 8$ & $4.7607 \times 10^{-3}$ & 1.9136 & $8.6408 \times 10^{-3}$ & 1.9935 & $1.4560 \times 10^{-2}$ \\
$1 / 16$ & $1.2281 \times 10^{-3}$ & 1.9547 & $2.1630 \times 10^{-3}$ & 1.9981 & $3.7084 \times 10^{-3}$ \\
$1 / 32$ & $3.1192 \times 10^{-4}$ & 1.9772 & $5.4001 \times 10^{-4}$ & 2.0020 & $9.2814 \times 10^{-4}$ \\
$1 / 64$ & $7.8041 \times 10^{-5}$ & 1.9989 & $1.2619 \times 10^{-4}$ & 2.0973 & $2.2497 \times 10^{-4}$ \\
\hline
\end{tabular}

TABLE 6: The spatial convergence rate can achieve to $\mathcal{O}\left(h^{k+1}\right) ; \alpha=0.5$ and $T=1$.

\begin{tabular}{lccccc}
\hline$h$ & $\beta=1.02$ & Rate & $\beta=1.4$ & Rate & $\beta=1.9$ \\
\hline $1 / 4$ & $4.5016 \times 10^{-2}$ & - & $5.1678 \times 10^{-3}$ & - & $8.4526 \times 10^{-3}$ \\
$1 / 8$ & $1.1740 \times 10^{-2}$ & 1.9389 & $1.3028 \times 10^{-3}$ & 1.9879 & $2.1608 \times 10^{-3}$ \\
$1 / 16$ & $3.0014 \times 10^{-3}$ & 1.9678 & $3.2606 \times 10^{-4}$ & 1.9984 & $5.4081 \times 10^{-4}$ \\
$1 / 32$ & $7.2299 \times 10^{-4}$ & 2.0536 & $7.7774 \times 10^{-5}$ & 2.0678 & 1.9678 \\
$1 / 64$ & $1.6926 \times 10^{-4}$ & 2.0947 & $1.8152 \times 10^{-5}$ & 2.0991 & 3.9984 \\
\hline
\end{tabular}

TABLE 7: The temporal convergence rate can arrive at $\mathcal{O}\left(\tau^{2-\alpha}\right) ; \beta=1.6$ and $T=1$.

\begin{tabular}{lccccc}
\hline$\tau$ & $\alpha=0.1$ & Rate & $\alpha=0.7$ & Rate & $\alpha=0.97$ \\
\hline $1 / 4$ & $6.0398 \times 10^{-3}$ & - & $5.5331 \times 10^{-3}$ & - & $8.9365 \times 10^{-3}$ \\
$1 / 8$ & $1.6957 \times 10^{-3}$ & 1.8326 & $2.3184 \times 10^{-3}$ & 1.2549 & $4.8416 \times 10^{-3}$ \\
$1 / 16$ & $4.6458 \times 10^{-4}$ & 1.8679 & $9.5726 \times 10^{-4}$ & 1.2762 & $2.5280 \times 10^{-3}$ \\
$1 / 32$ & $1.2436 \times 10^{-4}$ & 1.9014 & $3.8866 \times 10^{-4}$ & 1.3004 & 0.8842 \\
$1 / 64$ & $3.3314 \times 10^{-5}$ & 1.9003 & $1.5764 \times 10^{-4}$ & 1.3018 & 0.9375 \\
\hline
\end{tabular}


TABLE 8: The temporal convergence rate can arrive at $\mathcal{O}\left(\tau^{2-\alpha}\right) ; \alpha=0.1$ and $T=1$.

\begin{tabular}{lcccccc}
\hline$\tau$ & $\beta=1.2$ & Rate & $\beta=1.6$ & Rate & $\beta=1.99$ & Rate \\
\hline $1 / 4$ & $3.2985 \times 10^{-3}$ & - & $7.0233 \times 10^{-3}$ & - & $6.4021 \times 10^{-3}$ \\
$1 / 8$ & $8.9844 \times 10^{-4}$ & 1.8763 & $1.8923 \times 10^{-3}$ & 1.8920 & $1.7360 \times 10^{-3}$ \\
$1 / 16$ & $2.4326 \times 10^{-4}$ & 1.8849 & $5.1141 \times 10^{-4}$ & 1.8876 & $4.6633 \times 10^{-4}$ \\
$1 / 32$ & $6.5050 \times 10^{-5}$ & 1.9029 & $1.3624 \times 10^{-4}$ & 1.9083 & $1.2490 \times 10^{-4}$ \\
$1 / 64$ & $1.7311 \times 10^{-5}$ & 1.9098 & $3.5915 \times 10^{-5}$ & 1.9235 & $3.3368 \times 10^{-5}$ \\
\hline
\end{tabular}

TABLE 9: The spatial convergence rate can achieve to $\mathcal{O}\left(h^{k+1}\right) ; \beta=1.6$ and $T=1$.

\begin{tabular}{lccccc}
\hline$h$ & $\alpha=1.05$ & Rate & $\alpha=1.6$ & Rate & $\alpha=1.999$ \\
\hline $1 / 4$ & $3.8952 \times 10^{-2}$ & - & $7.9493 \times 10^{-2}$ & - & $5.0248 \times 10^{-2}$ \\
$1 / 8$ & $9.9577 \times 10^{-3}$ & 1.9678 & $2.1840 \times 10^{-2}$ & 1.8638 & $1.3661 \times 10^{-2}$ \\
$1 / 16$ & $2.5203 \times 10^{-3}$ & 1.9822 & $5.7575 \times 10^{-3}$ & 1.9235 & $3.5435 \times 10^{-3}$ \\
$1 / 32$ & $6.2991 \times 10^{-4}$ & 2.0004 & $1.4499 \times 10^{-3}$ & 1.9895 & $8.9173 \times 10^{-4}$ \\
$1 / 64$ & $1.5363 \times 10^{-4}$ & 2.0356 & $3.5356 \times 10^{-4}$ & 2.0359 & $2.2245 \times 10^{-4}$ \\
\hline
\end{tabular}

TABLE 10: The spatial convergence rate can achieve to $\mathcal{O}\left(h^{k+1}\right) ; \alpha=1.3$ and $T=1$.

\begin{tabular}{lccccc}
\hline$h$ & $\beta=1.05$ & Rate & $\beta=1.5$ & Rate & $\beta=1.9$ \\
\hline $1 / 4$ & $6.0285 \times 10^{-2}$ & - & $4.9039 \times 10^{-2}$ & - & $6.7648 \times 10^{-2}$ \\
$1 / 8$ & $1.6179 \times 10^{-2}$ & 1.8976 & $1.3190 \times 10^{-2}$ & 1.8944 & $1.7680 \times 10^{-2}$ \\
$1 / 16$ & $4.2071 \times 10^{-3}$ & 1.9433 & $3.4816 \times 10^{-3}$ & 1.9217 & $4.5017 \times 10^{-3}$ \\
$1 / 32$ & $1.0652 \times 10^{-3}$ & 1.9816 & $8.8475 \times 10^{-4}$ & 1.9764 & 1.9359 \\
$1 / 64$ & $2.6718 \times 10^{-4}$ & 1.9953 & $2.2166 \times 10^{-4}$ & 1.9969 & $2.6435 \times 10^{-3}$ \\
\hline
\end{tabular}

TABLE 11: The temporal convergence rate can reach to $\mathcal{O}\left(\tau^{3-\alpha}\right) ; \beta=1.5, C^{\prime}=0.5$, and $T=1$.

\begin{tabular}{lccccc}
\hline$\tau$ & $\alpha=1.1$ & Rate & $\alpha=1.3$ & Rate & $\alpha=1.99$ \\
\hline $1 / 4$ & $3.7566 \times 10^{-2}$ & - & $3.2989 \times 10^{-2}$ & - & $3.0408 \times 10^{-3}$ \\
$1 / 8$ & $1.0324 \times 10^{-2}$ & 1.8634 & $1.0920 \times 10^{-2}$ & 1.5949 & $1.6243 \times 10^{-3}$ \\
$1 / 16$ & $2.6767 \times 10^{-3}$ & 1.9475 & $3.5105 \times 10^{-3}$ & 1.6373 & $8.3049 \times 10^{-4}$ \\
$1 / 32$ & $6.7308 \times 10^{-4}$ & 1.9916 & $1.0781 \times 10^{-3}$ & 1.7032 & 0.9046 \\
$1 / 64$ & $1.6666 \times 10^{-4}$ & 2.0138 & $3.2889 \times 10^{-4}$ & 1.7128 & 0.9678 \\
\hline
\end{tabular}

TABLE 12: The temporal convergence rate can reach to $\mathcal{O}\left(\tau^{3-\alpha}\right) ; \alpha=1.8, C^{\prime}=0.6$, and $T=1$.

\begin{tabular}{lccccc}
\hline$\tau$ & $\beta=1.15$ & Rate & $\beta=1.49$ & Rate & $\beta=1.999$ \\
\hline $1 / 4$ & $2.2799 \times 10^{-3}$ & - & $3.6088 \times 10^{-3}$ & - & $6.5082 \times 10^{-3}$ \\
$1 / 8$ & $1.0716 \times 10^{-3}$ & 1.0891 & $1.6547 \times 10^{-3}$ & 1.1249 & $3.0294 \times 10^{-3}$ \\
$1 / 16$ & $4.9550 \times 10^{-4}$ & 1.1129 & $7.2342 \times 10^{-4}$ & 1.1937 & $1.3576 \times 10^{-3}$ \\
$1 / 32$ & $2.1798 \times 10^{-4}$ & 1.1847 & $3.1736 \times 10^{-4}$ & 1.1887 & $5.9499 \times 10^{-4}$ \\
$1 / 64$ & $9.4494 \times 10^{-5}$ & 1.2059 & $1.3783 \times 10^{-4}$ & 1.2032 & $2.5780 \times 10^{-4}$ \\
\hline
\end{tabular}

$$
\begin{cases}\frac{\partial^{\alpha} u(x, t)}{\partial t^{\alpha}}+u(x, t) \frac{\partial u(x, t)}{\partial x}-a(x) \varepsilon\left(-(-\Delta)^{\beta / 2}\right) u(x, t)=h(x, t), & x \in \Omega, t \in[0, T], \\ u(x, 0)=x^{3}, & u_{t}(x, 0)=-4 x^{3}, x \in \Omega, \\ u(0, t)=0, & u(1, t)=(2 t-1)^{2}, x \in \partial \Omega,\end{cases}
$$

where $\Omega=[0,1], T=1,1<\alpha<2,1<\beta<2, a(x)=1, \epsilon=1$; we choose the exact solution of the equation as $u(x, t)=(2 t-1)^{2} x^{3}$, and $h(x, t)$ is from equation (90).
Similar to the nonlinear subdiffusion model, we also choose alternating direction flux for the linear term and LaxFriedrichs flux for the nonlinear term and select the 
appropriate time step size $\tau$ such that the time discretization errors are negligible as compared to the spatial errors. The $L^{2}$ error and convergence rate in spatial direction are indicated in Tables 9 and 10, in which one of the parameters $\beta$ (or $\alpha$ ) is fixed and the other parameter $\alpha$ (or $\beta$ ) is changed. As can be seen from the data in tables, both schemes have optimal convergence rate $\mathcal{O}\left(h^{k+1}\right)$ in the spatial direction; this conclusion is consistent with the theoretical results predicted in Theorem 4 . When $h=\mathrm{Cl} \tau$ $(C l)$, we choose the time step size sequence as $\tau=1 / 2^{i},(i=2, \cdots, 6)$ and fix $\beta$ (or $\alpha$ ), and then change $\alpha(\operatorname{or} \beta)$; the results in Tables 11 and 12 show that when $1<\alpha<2$, the convergence rate in time direction for the time-space fractional superdiffusion can reach to $\mathcal{O}\left(\tau^{3-\alpha}\right)$ and tends to 1 when $\alpha \longrightarrow 2$. These results are consistent with the theoretical consequence proved in Theorem 4.

\section{Conclusions}

In this paper, we proposed two fully discrete local discontinuous Galerkin schemes for solving nonlinear timespace fractional subdiffusion/superdiffusion equations, respectively. Through carefully choosing the numerical flux on boundary terms and making a concrete theoretical analysis, we proved that our numerical schemes are unconditional stable and convergent under the $L^{2}$ norm. According to the numerical results, we observe that when $0<\alpha<1$, a convergence rate $\mathcal{O}\left(\tau^{2-\alpha}+h^{k+1}\right)$ can be achieved. And when $1<\alpha<2$ and if we choose the space step size $h$ and the time step size $\tau$ satisfies the relationship $h=C / \tau(C /$ is a constant), the convergence order of the proposed schemes can approach to $\mathcal{O}\left(\tau^{3-\alpha}+h^{k+1}\right)$, which is consistent with the theoretical consequence proved in our theorems. The numerical results show that the fully discrete local discontinuous Galerkin (LDG) approximation is an effective and powerful method for solving the time-space fractional subdiffusion/superdiffusion equations.

\section{Data Availability}

The data used to support the findings of this study are included within the article.

\section{Conflicts of Interest}

The authors declare that there are no conflicts of interest regarding the publication of this paper.

\section{Acknowledgments}

This work was supported by the Natural Science Foundation of Guangdong Province of China (2018A030310038) and the talent project of Huizhou University of China (2017JB010) and the Doctor Scientific Research Foundation of Chongqing University of Posts and Telecommunications (A2018-20).

\section{References}

[1] K. S. Miller and B. Ross, An Introduction to the Fractional Calculus and Fractional Differential Equations, Wiley, New York, NY, USA, 1993.

[2] I. Podlubny, "Fractional differential equations," SIAM Review, vol. 42 , pp. 766-768, 2000.

[3] A. A. Kilbas, H. M. Srivastava, and J. J. Trujillo, Theory and Applications of Fractional Differential Equations, Elsevier, Amsterdam, Netherlands, 2006.

[4] R. Metzler and J. Klafter, "The random walk's guide to anomalous diffusion: a fractional dynamics approach," Physics Reports, vol. 339, no. 1, pp. 1-77, 2000.

[5] R. Gorenflo, F. Mainardi, D. Moretti, G. Pagnini, and P. Paradisi, "Discrete random walk models for space-time fractional diffusion," Chemical Physics, vol. 284, no. 1-2, pp. 521-541, 2002.

[6] Y.-M. Wang, "A compact finite difference method for solving a class of time fractional convection-subdiffusion equations," BIT Numerical Mathematics, vol. 55, no. 4, pp. 1187-1217, 2015.

[7] C.-C. Ji and Z.-Z. Sun, "A high-order compact finite difference scheme for the fractional sub-diffusion equation," Journal of Scientific Computing, vol. 64, no. 3, pp. 959-985, 2015.

[8] F. Zeng, Z. Zhang, and G. E. Karniadakis, "Fast difference schemes for solving high-dimensional time-fractional subdiffusion equations," Journal of Computational Physics, vol. 307, pp. 15-33, 2016.

[9] G.-H. Gao and Z.-Z. Sun, "A compact finite difference scheme for the fractional sub-diffusion equations," Journal of Computational Physics, vol. 230, no. 3, pp. 586-595, 2011.

[10] Y.-N. Zhang, Z.-Z. Sun, and H.-W. Wu, "Error estimates of crank-nicolson-type difference schemes for the subdiffusion equation," SIAM Journal on Numerical Analysis, vol. 49, no. 6, pp. 2302-2322, 2011.

[11] Z. Wang and S. Vong, "Compact difference schemes for the modified anomalous fractional sub-diffusion equation and the fractional diffusion-wave equation," Journal of Computational Physics, vol. 277, pp. 1-15, 2014.

[12] B. T. Jin, B. Y. Li, and Z. Zhou, "An analysis of the Crank-Nicolson method for subdiffusion," IMA Journal of Numerical Analysis, vol. 38, no. 1, pp. 1-23, 2017.

[13] S. Shen, F. Liu, and V. Anh, "Numerical approximations and solution techniques for the space-time Riesz-Caputo fractional advection-diffusion equation," Numerical Algorithms, vol. 56, no. 3, pp. 383-403, 2011.

[14] A. Saadatmandi, M. Dehghan, and M.-R. Azizi, "The SincLegendre collocation method for a class of fractional convection-diffusion equations with variable coefficients," Communications in Nonlinear Science and Numerical Simulation, vol. 17, no. 11, pp. 4125-4136, 2012.

[15] Q. Liu, F. Liu, I. Turner, V. Anh, and Y. T. Gu, "A RBF meshless approach for modeling a fractal mobile/immobile transport model," Applied Mathematics and Computation, vol. 226, no. 1, pp. 336-347, 2014.

[16] Y. Jiang and J. Ma, "High-order finite element methods for time-fractional partial differential equations," Journal of Computational and Applied Mathematics, vol. 235, no. 11, pp. 3285-3290, 2011.

[17] F. Zeng, C. Li, F. Liu, and I. Turner, "The use of finite difference/element approaches for solving the time-fractional subdiffusion equation," SIAM Journal on Scientific Computing, vol. 35, no. 6, pp. A2976-A3000, 2013. 
[18] X. Hu, H.-L. Liao, F. Liu, and I. Turner, "A center box method for radially symmetric solution of fractional subdiffusion equation," Applied Mathematics and Computation, vol. 257, pp. 467-486, 2015.

[19] A. Bhrawy, D. Baleanu, and F. Mallawi, "A new numerical technique for solving fractional sub-diffusion and reaction sub-diffusion equations with a non-linear source term," Thermal Science, vol. 19, no. 1, pp. 25-34, 2015.

[20] Y. Du, Y. Liu, H. Li, Z. Fang, and S. He, "Local discontinuous Galerkin method for a nonlinear time-fractional fourth-order partial differential equation," Journal of Computational Physics, vol. 344, pp. 108-126, 2017.

[21] T. Aboelenen, "Local discontinuous Galerkin method for distributed-order time and space-fractional convection-diffusion and Schrödinger-type equations," Nonlinear Dynamics, vol. 92, no. 2, pp. 395-413, 2018.

[22] Y. Liu, M. Zhang, H. Li, and J. Li, "High-order local discontinuous Galerkin method combined with WSGD-approximation for a fractional subdiffusion equation," Computers \& Mathematics with Applications, vol. 73, no. 6, pp. 1298-1314, 2017.

[23] C. Li, Z. Zhao, and Y. Chen, "Numerical approximation of nonlinear fractional differential equations with subdiffusion and superdiffusion," Computers \& Mathematics with Applications, vol. 62, no. 3, pp. 855-875, 2011.

[24] M. Qiu, L. Mei, and D. Li, "Fully discrete local discontinuous Galerkin approximation for time-space fractional subdiffusion/superdiffusion equations," Advances in Mathematical Physics, vol. 2017, Article ID 4961797, 20 pages, 2017.

[25] J. Klafter, B. S. White, and M. Levandowsky, "Microzooplankton feeding behavior and the Lévy walk," Lecture Notes in Biomathematics, pp. 281-296, Springer-Verlag, Berlin, Germany, 1990.

[26] G. M. Zaslavsky, "Chaos, fractional kinetics, and anomalous transport," Physics Reports, vol. 371, no. 6, pp. 461-580, 2017.

[27] B. Cockburn and C.-W. Shu, "The local discontinuous Galerkin method for time-dependent convection-diffusion systems," SIAM Journal on Numerical Analysis, vol. 35, no. 6, pp. 2440-2463, 1998.

[28] B. Cockburn and C.-W. Shu, "Runge-Kutta discontinuous Galerkin methods for convection-dominated problems," Journal of Scientific Computing, vol. 16, no. 3, pp. 173-261, 2001.

[29] Y. Xu and C.-W. Shu, "Local discontinuous Galerkin methods for high-order time-dependent partial differential equations," Communications in Computational Physics, vol. 7, no. 1, pp. 1-46, 2010.

[30] W. Deng and J. S. Hesthaven, "Local discontinuous Galerkin methods for fractional ordinary differential equations," BIT Numerical Mathematics, vol. 55, no. 4, pp. 967-985, 2015.

[31] L. Guo, Z. Wang, and S. Vong, "Fully discrete local discontinuous Galerkin methods for some time-fractional fourthorder problems," International Journal of Computer Mathematics, vol. 93, no. 10, pp. 1665-1682, 2015.

[32] L. Wei and Y. He, "Analysis of a fully discrete local discontinuous Galerkin method for time-fractional fourth-order problems," Applied Mathematical Modelling, vol. 38, no. 4, pp. 1511-1522, 2014.

[33] Q. Xu and Z. S. Zheng, "Discontinuous Galerkin method for time fractional diffusion equation," Journal of Information and Computational Science, vol. 10, no. 11, pp. 3253-3264, 2013.
[34] Q. Xu and J. S. Hesthaven, "Discontinuous Galerkin method for fractional convection-diffusion equations," SIAM Journal on Numerical Analysis, vol. 52, no. 1, pp. 405-423, 2014.

[35] V. J. Ervin and J. P. Roop, "Variational formulation for the stationary fractional advection dispersion equation," $\mathrm{Nu}$ merical Methods for Partial Differential Equations, vol. 22, no. 3, pp. 558-576, 2006.

[36] J. J. Zhao, J. Y. Xiao, and Y. Xu, "A finite element method for the multiterm time-space Riesz fractional advection-diffusion equations in finite domain," Abstract and Applied Analysis, vol. 2013, Article ID 868035, 16 pages, 2013.

[37] B. Cockburn, G. Kanschat, I. Perugia, and D. Schötzau, "Superconvergence of the local discontinuous Galerkin method for elliptic problems on Cartesian grids," SIAM Journal on Numerical Analysis, vol. 39, no. 1, pp. 264-285, 2001.

[38] Y. Lin and C. Xu, "Finite difference/spectral approximations for the time-fractional diffusion equation," Journal of Computational Physics, vol. 225, no. 2, pp. 1533-1552, 2007.

[39] L. Wei, X. Zhang, and Y. He, "Analysis of a local discontinuous Galerkin method for time-fractional advection-diffusion equations," International Journal of Numerical Methods for Heat \& Fluid Flow, vol. 23, no. 4, pp. 634-648, 2013.

[40] J. S. Hesthaven and T. Warburton, Nodal Discontinuous Galerkin Methods: Algorithms, Analysis and Applications, Springer-Verlag, Berlin, Germany, 2008.

[41] Y. Xu and C.-W. Shu, "Error estimates of the semi-discrete local discontinuous Galerkin method for nonlinear convection-diffusion and KdV equations," Computer Methods in Applied Mechanics and Engineering, vol. 196, no. 37-40, pp. 3805-3822, 2007.

[42] Q. Zhang and C.-W. Shu, "Error estimates to smooth solutions of Runge-Kutta discontinuous Galerkin methods for scalar conservation laws," SIAM Journal on Numerical Analysis, vol. 42, no. 2, pp. 641-666, 2005. 